RAA Vol. No.,

http://www.raa-journal.org http://www.iop.org/journals/raa

Research in

$\boldsymbol{A}$ stronomy and

Astrophysics

Key words: galaxies: star clusters: general — galaxies: star formation — stars: rotation

\title{
Stellar populations in star clusters
}

\author{
Cheng-Yuan $\mathrm{Li}^{1,2}$, Richard de Grijs ${ }^{3,4}$ and Li-Cai Deng ${ }^{5}$ \\ ${ }^{1}$ Department of Physics and Astronomy, Macquarie University, North Ryde, NSW 2109, \\ Australia; chengyuan.li@mq.edu.au \\ 2 Purple Mountain Observatory, Chinese Academy of Sciences, Nanjing 210008, China \\ 3 Kavli Institute for Astronomy \& Astrophysics and Department of Astronomy, Peking University, \\ Yi He Yuan Lu 5, Hai Dian District, Beijing 100871, China \\ ${ }^{4}$ International Space Science Institute-Beijing, 1 Nanertiao, Zhongguancun, Hai Dian District, \\ Beijing 100190, China \\ ${ }^{5}$ Key Laboratory for Optical Astronomy, National Astronomical Observatories, Chinese Academy \\ of Sciences, 20A Datun Road, Chaoyang District, Beijing 100012, China
}

\begin{abstract}
Stellar populations contain the most important information about star cluster formation and evolution. Until several decades ago, star clusters were believed to be ideal laboratories for studies of simple stellar populations (SSPs). However, discoveries of multiple stellar populations in Galactic globular clusters have expanded our view on stellar populations in star clusters. They have simultaneously generated a number of controversies, particularly as to whether young star clusters may have the same origin as old globular clusters. In addition, extensive studies have revealed that the SSP scenario does not seem to hold for some intermediate-age and young star clusters either, thus making the origin of multiple stellar populations in star clusters even more complicated. Stellar population anomalies in numerous star clusters are well-documented, implying that the notion of star clusters as true SSPs faces serious challenges. In this review, we focus on stellar populations in massive clusters with different ages. We present the history and progress of research in this active field, as well as some of the most recent improvements, including observational results and scenarios that have been proposed to explain the observations. Although our current ability to determine the origin of multiple stellar populations in star clusters is unsatisfactory, we propose a number of promising projects that may contribute to a significantly improved understanding of this subject.
\end{abstract}

\section{INTRODUCTION}

Star clusters are the basic units of star formation (Lada \& Lada 2003): almost all stars form in clustered environments. Current consensus on the formation of star clusters suggests that most stars form tracing the turbulent structure of the interstellar medium and in an initially supervirial state. Within an extremely short period (about one crossing time), the initially turbulent, 'fractal' structures will collapse into bound clusters (Bonnell et al.2008, Allison et al. 2009; Girichidis et al.|2012; Moeckel \& Burkert 2015). Subsequently, a large proportion will gradually dissipate into the galactic field 
(de Grijs 2010). Understanding the stellar populations of star clusters is, therefore, of fundamental importance for understanding many astrophysical processes, including star cluster formation and evolution, the chemical evolution of Galactic stellar populations, as well as the stellar dynamics in star clusters.

The 'simple stellar population' (SSP) scenario is the assumption that stars in a star cluster all originate from a common progenitor giant molecular cloud (GMC), during the same era, and thus they would share similar metallicities. It has been confirmed that the initial star-forming process in star clusters approximately resembles a single burst (Cabrera-Ziri et al.2014). The combination of gas expulsion owing to energetic photons ejected by the most massive first-generation stars and the strong stellar winds triggered by the first batch of Type II supernovae will quickly exhaust all of the gas in the GMC, thus quenching the star- and cluster-forming process (Bastian \& Strader 2014). The nature of most open clusters (OCs) and young massive clusters (YMCs) has been confirmed as resembling SSPs.

During the last few decades, observations have revealed the presence of multiple stellar populations in Galactic globular clusters (GCs). The observational evidence can be classified into photometric and spectroscopic evidence. The former refers to the fact that the photometric color-magnitude diagrams (CMDs) of some GCs display multiple distinct features in or along their main sequences (MSs; e.g., NGC 2808: Piotto et al.2007), their subgiant branches (SGBs; e.g., 47 Tuc: Anderson et al. 2009), their red-giant branches (RGBs; e.g., NGC 288: Piotto et al. 2013), or even in their horizontal branches (HBs; e.g., NGC 2808: Bedin et al. 2000). Sometimes, individual GCs even display a combination of such multiple features. In the photometric CMDs, these features can be explained as the result of a diversity of ages, helium abundances, and metallicities. Since SSP stars formed at approximately the same time from a common molecular cloud, variations in age, helium, and metal abundance hence unambiguously reflect the occurrence of more than a single star-forming episode during their host clusters' evolution.

GCs have also been subject to intense scrutiny based on spectroscopic analyses, which have revealed star-to-star chemical dispersions of specific elements. Cohen et al. (1978) first noted that the $\mathrm{Na}$ abundance variations of RGB stars in the GCs M3 and M13 exceeded the observational errors. Her pioneering work has stood the test of time: subsequent measurements of Galactic GCs have uncovered the well-known $\mathrm{Na}-\mathrm{O}$ anticorrelation, where oxygen-depleted stars have higher sodium abundance (Carretta et al. 2004a, Gratton et al. 2004, Carretta et al. 2009). Another well-studied Galactic GC relationship is the $\mathrm{Mg}-\mathrm{Al}$ anticorrelation, which is most easily seen in intermediatemetallicity clusters (e.g., M13; Shetrone 1996). The large scatter in the abundances of some specific elements is not expected if these GCs were SSPs. A straightforward explanation of the scatter properties is that there has been secondary star formation, fueled by abundance-enhanced material. In this review we will not discuss the chemical evolution of stellar populations in depth, since this aspect was introduced in great detail in the review of Gratton et al. (2004).

A key problem associated with the stellar populations in star clusters relates to an important open question: do GCs have the same origin as OCs and YMCs? It seems that the latter, very young objects may encounter significant difficulties to survive to the cosmic age ( $\geq 10 \mathrm{Gyr}$ ) owing to a range of effects, including internal two-body relaxation, which causes most stars to 'evaporate' from the cluster (Spitzer 1987). Only those clusters that initially contained at least $10^{5}$ stars can avoid disruption within $10 \mathrm{Gyr}$ (Portegies Zwart et al. 2010). On the other hand, in terms of their initial masses, only young star clusters with masses between $10^{5} M_{\odot}$ and $10^{6} M_{\odot}$ have the capacity to capture their initial runaway gas. Because the subsequent Type II supernovae explosions would further accelerate the runway gas flows, the mass threshold for a YMC to capture its initial runaway gas would increase dramatically. If a star cluster can not marshall additional gas reserves, its starforming process will cease rapidly. As regards the stellar populations in star clusters, almost all observed OCs and YMCs will fail to generate multiple stellar populations, which, however, is a common feature of most observed GCs (see Li 2015, his Chapter 4). 
This review is organized as follows. In Section 2 we show that the SSP approximation is not a tentative assumption but based on convincing evidence. Readers will realize why the presence of multiple stellar populations in GCs (and also in some young and intermediate-age clusters) is a problem. In Section 3 we discuss observational results of stellar populations in star clusters with different ages. In Section 4 we compare and contrast currently popular scenarios that aim at explaining the origin of multiple stellar populations; their advantages and disadvantages are also discussed. We next discuss, in Section 5, how one can go about examining some of these compelling and controversial scenarios. A brief summary is given in the concluding section (Section 6).

\section{THOUGHTS ON STELLAR POPULATIONS IN STAR CLUSTERS}

The first issue confronting any fundamental discussion of stellar populations is the star-formation mode in star clusters. Theoretically, except for the most massive and most compact star clusters, stars in a star cluster should form in single-burst mode: the star-formation process in a star cluster will cease rapidly after formation of the first-generation stars owing to the quick exhaustion of the initial gas, which is mainly caused by stellar wind-induced mass loss (e.g., energetic photons ejected by the most massive stars; Longmore et al. 2014) and supernova explosions. The typical escape velocity should be comparable to the sound speed in ionized hydrogen (HII) gas ( $\sim 10 \mathrm{~km} \mathrm{~s}^{-1}$; Banerjee \& Kroupa 2015). For some massive clusters, gas expulsion may be dominated by radiation pressure, and therefore their escape velocities could even exceed $10 \mathrm{~km} \mathrm{~s}^{-1}$ (Krumholz \& Matzner 2009). The timescale of gas expulsion driven by these initial stellar winds is very short, usually less than or comparable to the cluster's crossing time $(\sim 1 \mathrm{Myr})$. If a single supernova explosion from the rapid evolution of the most massive star occurs at this early stage, this timescale may even be close to zero, i.e., a cluster will be exposed (devoid of gas) almost immediately after birth.

Even if a cluster can retain its gas until the end of the initial gas expulsion phase ( $\leq 1 \mathrm{Myr}$ ), during the period from $3 \mathrm{Myr}$ to $10 \mathrm{Myr}$ most O-type stars $\left(\sim 16 M_{\odot}\right.$ to $\left.25 M_{\odot}\right)$ evolve off the MS. A $15 M_{\odot}$ star could eject $3 \times 10^{50}$ erg into the interstellar medium over the period of $0.1 \mathrm{Myr}$, which is sufficient to unbind $10^{4} M_{\odot}$ gas within a 1 pc region (Baumgardt \& Kroupa 2007). Therefore, a large fraction of residual gas in a cluster with an age between $3 \mathrm{Myr}$ an $10 \mathrm{Myr}$ is not expected. During this phase, Type II supernova explosions will occur frequently. These multiple supernova explosions will be the 'death blow' to any residual gas remaining in the cluster. Supernova explosions will expel all stellar material at a velocity of up to $3 \times 10^{4} \mathrm{~km} \mathrm{~s}^{-1}$ (10\% of the speed of light), driving a shock wave through the interstellar medium of their host star clusters. Such a shock wave will accelerate all remaining gas to a velocity of several $100 \mathrm{~km} \mathrm{~s}^{-1}$. Calculations have shown that only clusters that have formed from the initial condensations with masses of $10^{7} M_{\odot}$ to $10^{8} M_{\odot}$ can survive the first series of supernova explosions (Shustov \& Wiebe 2000).

Because the initial star-formation process ceases very quickly, the age range of the firstgeneration stars would thus be constrained to a timescale of several million years. This timescale, compared with the typical ages of most GCs ( $10 \mathrm{Gyr})$ and YMCs $(10 \mathrm{Myr}-100 \mathrm{Myr})$, is indeed very short.

Stars that are less massive than $\sim 8 M_{\odot}$ will undergo the post-RGB and asymptotic giant branch (AGB) phases, when they evolve to their final evolutionary stages. The ages of their host star clusters must be at least $30 \mathrm{Myr}$, which is already equivalent to the typical age of most YMCs. These intermediate-mass AGB stars will deposit most of their stellar material into the interstellar medium, which is thought to be important for secondary star formation: ejected stellar material from AGB stellar winds forms the building materials of newly born stars, possibly leading to the formation of multiple stellar populations with enhanced chemical abundances, at least compared with the initial stellar generation in the cluster. Many models aim at explaining the observed multiple-stellar populations in GCs are based on this type of 'self-enrichment' scenario (i.e., Ventura \& D'Antona 2009. Valcarce \& Catelan 2011). However, since in most GCs the observed secondary stellar generation 
has a comparable total mass to the first stellar generation, to explain the observed high fractions of secondary stellar generations, these scenarios have to assume extremely high cluster masses at early times (about 10 to 100 times their current masses). In addition, the velocity of the AGB stellar winds is comparable to the initial O-type stars' stellar winds, which are $\sim 10 \mathrm{~km} \mathrm{~s}^{-1}$ to $100 \mathrm{~km} \mathrm{~s}^{-1}$. Because initial gas expulsion will cause expansion of the cluster to a less compact configuration and leads to the loss of a fraction of the initial cluster mass, an exposed cluster is unlikely able to retain these intermediate-mass AGB stellar winds.

Mass loss caused by evaporation will further complicate the estimates (McLaughlin \& Fall 2008). The amount of mass lost through this mechanism can be calculated as

$$
\dot{m} \approx 1100\left(\frac{\rho_{\mathrm{h}}}{M_{\odot} \mathrm{pc}^{-3}}\right)^{-1 / 2} M_{\odot} \mathrm{Gyr}^{-1}
$$

where $\rho_{\mathrm{h}}$ is the gas density inside the cluster's half-mass radius, $r_{\mathrm{h}}$. Assuming a typical GC age of 12 Gyr, most GCs should initially have been 10 to 15 times more massive than their current mass, indicating a strong capacity to retain their initial runaway gas. However, for OCs and YMCs, most of which are younger than $\sim 100 \mathrm{Myr}$, the expected amount of mass loss is much smaller. Therefore, their initial masses do not reach the mass threshold for capturing runaway gas.

Assuming that the average half-mass radius of young clusters is $\sim 1 \mathrm{pc}$, we can evaluate the escape velocity (Georgiev et al.2009),

$$
v_{\mathrm{esc}}(t) \approx 0.1 \sqrt{\frac{M_{\mathrm{cl}}(t)}{r_{\mathrm{h}}(t)}} \quad \mathrm{km} \mathrm{s}^{-1} .
$$

It follows immediately that only for clusters with masses of $M_{\mathrm{cl}}=10^{4} M_{\odot}$ to $10^{6} M_{\odot}$, the initial runaway stellar material will be retained in their gravitational potential wells. In Fig. 1] we present the minimum mass for clusters to retain their initial runaway material as a function of their current mass for two different escape velocities: $10 \mathrm{~km} \mathrm{~s}^{-1}$ corresponds to the gas-expulsion velocity and the slowest AGB ejecta (top panel), while $100 \mathrm{~km} \mathrm{~s}^{-1}$ corresponds to the lowest escape velocity driven by Type II supernovae (bottom panel). Figure 1 shows that the OCs will fail to capture their initial runaway gas in both cases, while about half of the observed YMCs in the Milky Way will fail to capture the slowest AGB ejecta. For an escape velocity of $100 \mathrm{~km} \mathrm{~s}^{-1}$, all YMCs will lose their initial gas. This also holds for most GCs. Note that the escape velocity of $100 \mathrm{~km} \mathrm{~s}^{-1}$ is only a lower limit to the velocities generated by supernova explosions, so our estimates are conservative.

This is why star clusters are thought to approximate SSPs: during the initial phase $(\leq 1$ Myr), stellar feedback is sufficiently strong to unbind most residual gas from the protocluster; O-type stars will subsequently give rise to multiple supernova explosions (3 Myr to $10 \mathrm{Myr}$ ), cleaning out all remaining gas. Afterwards, a surviving cluster will become less compact and less massive, thus losing its capacity to retain additional gas.

Initial star-formation episodes in clusters cannot have lasted longer than the timescale of initial gas expulsion, i.e., the first-generation stars can only have been formed over a short timescale. In fact, the member stars of extremely young star clusters (with ages $\leq 3 \mathrm{Myr}$ ) are usually found in regions of their natal GMCs that are largely devoid of gas, while all YMCs older than several tens of millions of years are fully exposed.

Numerous studies of young star clusters have confirmed the SSP scenario: numerical simulations have shown that star formation proceeds in localized bursts within the cloud (Bate et al. 2003, Farias et al. 2015), after which gas expulsion will remove most of the initial molecular gas. Clusters will also lose many of their member stars at the same time (Baumgardt \& Kroupa 2007). Some nascent, extremely young clusters, commonly referred to as 'embedded clusters' (Bastian \& Goodwin 2006 

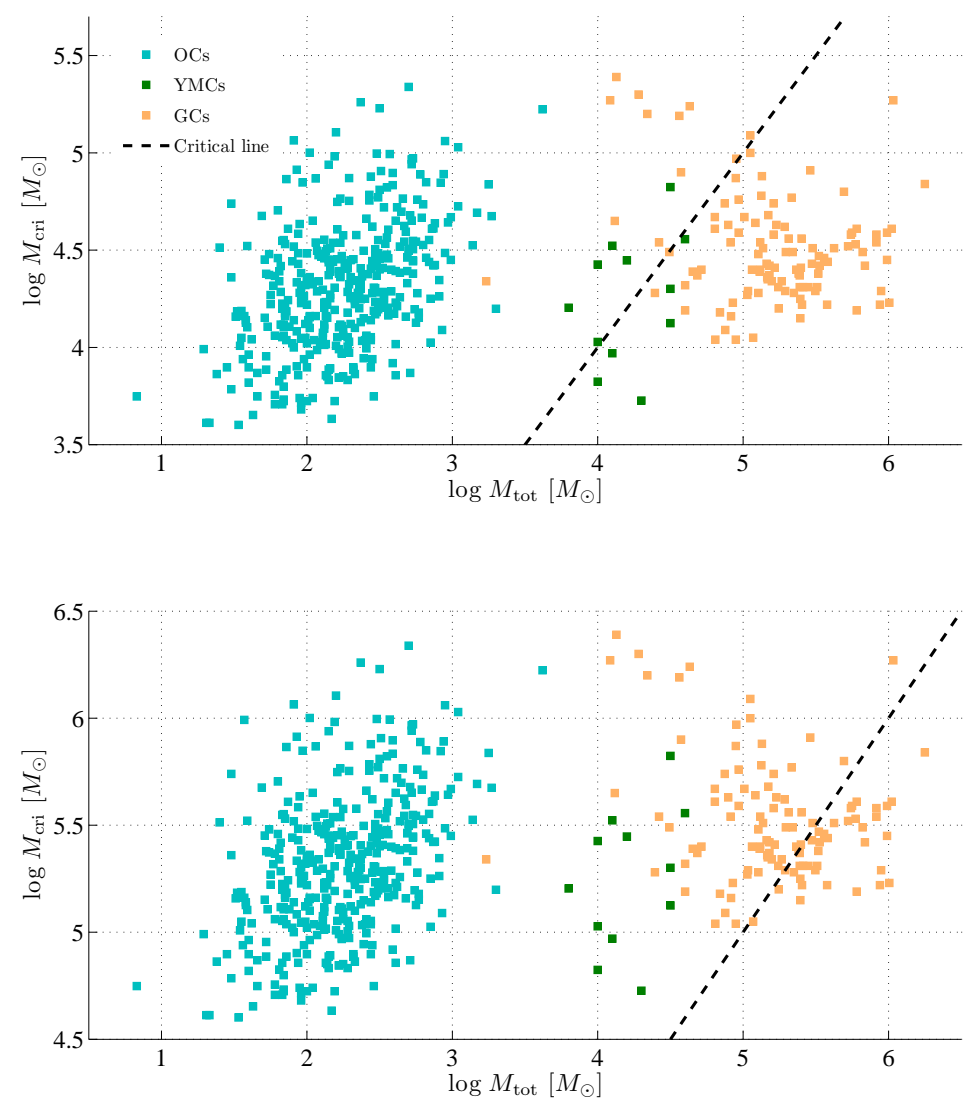

Fig. 1 Critical mass, $\log M_{\text {cri }}$, to capture the initial stellar winds of AGB stars for star clusters as a function of their actual mass, $\log M_{\text {tot }}$. Mint, dark green, and light orange rectangles: Galactic OCs, YMCs, and GCs, respectively. OC and YMC data are from Portegies Zwart et al. (2010, private communication). GC data are derived from (Djorgovski \& Meylan 1994, http://www.astro.caltech.edu/ ${ }^{2}$ george/glob/glob.data). The rectangles located above the critical (black dashed) lines are clusters which will not be able to retain their initial runaway material (based on their current masses). Escape velocities: (top) $10 \mathrm{~km} \mathrm{~s}^{-1}$; (bottom) $100 \mathrm{~km} \mathrm{~s}^{-1}$.

Davidge 2012; Lima et al. 2014), are indeed observed. Combined with observations of YMCs with ages of $\sim 100 \mathrm{Myr}$, several lines of evidence imply that there will be little residual gas for star formation in YMCs after the gas-expulsion period (Bastian et al. 2013; Bastian \& Strader 2014, Cabrera-Ziri et al.2015).

As discussed in Section 1, star clusters will lose stars through dynamical two-body relaxation. This disruption process is further accelerated by external tidal shocks. As a consequence, a star cluster's mass will continue to decrease during its entire evolution (if no additional merger or accretion 
events occur), which means that clusters can retain their initial gas only during the early stages of their evolution. However, detailed studies have confirmed that OCs and YMCs are SSPs, with rare exceptions, such as the cluster NGC 6791 (Geisler et al.2012).

The ubiquitous multiple stellar populations in GCs immediately pose a conundrum: How do these clusters trigger secondary star formation if most of their initial material would have been lost at an early stage? In fact, this issue relates to another key open question: Do GCs have the same origin as young star clusters? In Section 3 we introduce some relevant background to acquaint readers with the details of stellar populations in various types of star clusters.

\section{NOT-SO-SIMPLE STELLAR POPULATIONS IN STAR CLUSTERS}

\subsection{Stellar Populations in Young and Intermediate-age Clusters}

Spectroscopic analyses investigating star-to-star chemical variations provide evidence that OCs are chemically homogeneous (e.g., Shen et al. 2005, D’Orazi \& Randich 2009, Ting et al. 2012) — this seems to also hold for old OCs like NGC 188, see Norris \& Smith (1985) - thus confirming that their stars indeed formed coevally from a common GMC. This conclusion is also valid for stellar associations (e.g., De Silva et al. 2013) and star-forming regions (e.g., Nieva \& Simón-Díaz 2011), indicating a high degree of chemical homogeneity in the primordial GMCs.

The most direct method to examine if a group of stars in a star cluster are coeval is by exploring their CMD, since the CMD of an SSP can be described very well by a single isochrone. However, because of Galactic extinction, OC CMDs are usually messy, which renders explorations of the age distributions of their member stars difficult. Currently, measuring a single star's age is still challenging. No single method works well for a broad range of stellar types or for the full range in ages, but this may change in the next few years with improvements resulting from the technique of asteroseismology (Soderblom 2010).

Another promising approach to studying stellar ages is by measuring their surface lithium abundances, since the lithium abundance is suggested to decrease with increasing stellar age. Studies based on the Li clock surprisingly reveal that the ages of some stars seem to exceed their host OCs' isochronal ages (Duncan \& Jones 1983; Palla et al. 2007). However, it is unclear whether these exceptions can be made to resemble large-scale ongoing star formation. For instance, Sacco et al. (2007) showed that only three of their 59 stars analyzed in the young $\sigma$ Orionis cluster show Li depletion, and among the former only one star has a nuclear age that exceeds the isochronal age. Bik et al. (2014) analyzed the ages of the most massive O-type stars in the extremely young, massive cluster W3 Main (with an age of approximately 3-5 Myr). They found that star formation in W3 Main has lasted for about 2-3 Myr and is still ongoing.

In addition to the Li clock, which only works for individual stars, exploring the luminosity distribution of main-sequence turn-off (MSTO) stars provides an independent approach to test the SSP scenario. Although Galactic extinction makes studying the intrinsic magnitude spread of MSTO stars in OCs difficult, some exceptions exhibit robust statistical results. Based on a comparison of stellar isochrones and the observed stellar distribution in the CMD, Eggen (1998) found that the age ranges of the Hyades and Praesepe OCs seem to span several hundred million years, as implied by the apparent luminosity spread of MSTO stars. A similar luminosity dispersion has also been found in the Orion Nebula Cluster, for which an internal age spread on the order of 10 Myr was inferred (Palla \& Stahler 2000). More recent studies have found that NGC 1856, a 300 Myr-old Large Magellanic Cloud (LMC) cluster, displays an apparently extended MSTO (eMSTO) region (Milone et al. 2015a), which may indicate prolonged star formation, lasting $\sim 150 \mathrm{Myr}$. A recent study found that the 150 Myr-old YMC NGC 1850 also seems to harbor an eMSTO region (Bastian et al. 2016). Because both NGC 1850 and NGC 1856 reside in the LMC, they are not affected by significant extinction due to dust in the Galactic disk. The morphologies of the eMSTO regions in 
these YMCs may be a 'smoking gun,' suggesting that stars in star clusters may form continuously rather than in a single starburst event.

Such eMSTOs seem to be ordinary features of intermediate-age (1-2 Gyr-old) star clusters in the LMC and the Small Magellanic Cloud (SMC). For instance, Mackey \& Broby Nielsen (2007) found that the LMC cluster NGC 1846 shows a split in its MSTO region, which can be well described by two isochrones with ages of $1.5 \mathrm{Gyr}$ and $1.8 \mathrm{Gyr}$. Similar results have been found for the LMC clusters NGC 1783 and NGC 1806, with indications that their age dispersion may be at least $300 \mathrm{Myr}$ (Mackey et al.2008). Milone et al. (2009) studied 16 intermediate-age LMC clusters by exploring their MSTO regions, concluding that $70 \%$ have MSTOs that are inconsistent with the expectations from SSPs. Girardi et al. (2013) studied the SMC clusters NGC 411 and NGC 419. They found that their eMSTO regions are consistent with age spreads of $\sim 700 \mathrm{Myr}$, which may be the largest apparent age spreads known among all intermediate-age star clusters (but see Wu et al.2016). Similar results have also been found by Rubele et al. (2010, 2011). Girardi et al. (2009) even claimed the presence of dual red clumps (RCs) in NGC 419. Li et al. (2014a) analyzed the MSTO regions in the LMC clusters NGC 1831 and NGC 1868. They found that their implied internal age spreads are 280 Myr and $320 \mathrm{Myr}$, respectively, although the compact RC of NGC 1831 is inconsistent with such a large age spread.

The discovery of eMSTO regions in intermediate-age star clusters strongly challenges our understanding of star cluster formation and evolution. Various scenarios have been proposed to explain the observations. Goudfrooij et al. (2011, 2014) found a correlation between the widths of the MSTO regions and the early escape velocities in intermediate-age star clusters. They claimed a velocity threshold of $12-15 \mathrm{~km} \mathrm{~s}^{-1}$ for all clusters featuring eMSTOs, which is consistent with the escape velocity of gas expulsion and slow AGB stellar winds. This implies that the gas used for secondary star formation may have originated from intermediate-mass AGB stars or massive binary stars. However, since most of these star clusters are more massive than $10^{4} M_{\odot}$, their initial stellar populations should have contained many massive OB stars. It is then concerning that these OB stars did not expel the interstellar medium at very early stages through stellar feedback and supernova explosions. This scenario was challenged by Cabrera-Ziri et al. (2015), who carefully studied the stellar populations of NGC 7252-W3, a possible YMC candidate with an age of 570 Myr and an escape velocity of $193 \mathrm{~km} \mathrm{~s}^{-1}$. However, no evidence of an extended star-formation history (eSFH) was found. Bastian \& Strader (2014) searched for residual gas in 13 LMC and SMC YMCs with ages $\leq 300$ Myr. Their conclusion was, once again, negative: No residual gas was detected. In summary, most clusters are unable to accrete large gas reservoirs to support ongoing star formation. However, this does not mean that they will not be able to capture external gas after the initial gasexpulsion phase. Li et al. (2016b) found the clear presence of younger stellar populations in three intermediate-age star clusters, NGC 1783, NGC 1806, and NGC 411, which seem to have an external origin. These young stellar populations are tightly associated with SSP isochrones, which cannot be explained by contamination due to field stars spanning a range of ages and metallicities. In addition, the radial profiles of these stellar populations clearly peak in the clusters' central regions (for a more detailed rebuttal, see Section 4). They speculated that these star clusters may have accreted external gas to form new stars at a later stage, although the newly born stars only represent a minor fraction in mass.

As regards the eSFH problem, numerous debates now focus on whether other features of intermediate-age star clusters are also consistent with an eSFH. Li et al. (2014b) found that in the 1.7 Gyr-old cluster NGC 1651, despite exhibiting an eMSTO consistent with a 450 Myr age spread, the SGB morphology can only be reconciled with an age spread of $\leq 160 \mathrm{Myr}$. The same conclusion was reached by Bastian \& Niederhofer (2015) for NGC 1806 and NGC 1846. However, their results were criticized by Goudfrooij et al. (2015), who performed numerical simulations based on these clusters' physical parameters to show that the morphologies of their SGBs may still be consistent with significant age spreads. To explain the apparent inconsistencies between the widths of the SGBs 
and the stellar evolutionary models, they suggested stellar convective overshooting as a viable alternative solution. However, Li et al. (2016a) found a more conspicuously narrow SGB in NGC 411, which can only be explained by an SSP. This is in obvious contrast with the extent of its eMSTO region. They concluded that NGC 411 is more likely composed of a coeval stellar population rather than of multiple-aged stellar populations. Girardi et al.(2013) claimed that NGC 411 appears to have experienced an eSFH lasting $\sim 700 \mathrm{Myr}$, which immediately introduces an apparent discrepancy with respect to the observed SGB. On the other hand, Mucciarelli et al. (2014) studied eight giant stars in the cluster NGC 1806 and did not find any evidence of a possible $\mathrm{Na}-\mathrm{O}$ anticorrelation, common to almost all Galactic GCs. The discovery of Mucciarelli et al. (2014) indeed stands in marked contrast to the chemical variations present in GCs. In Fig. 2 we present the $\mathrm{Na}-\mathrm{O}$ diagram pertaining to the NGC 1806 stars (pink), compared with that of GCs (gray).

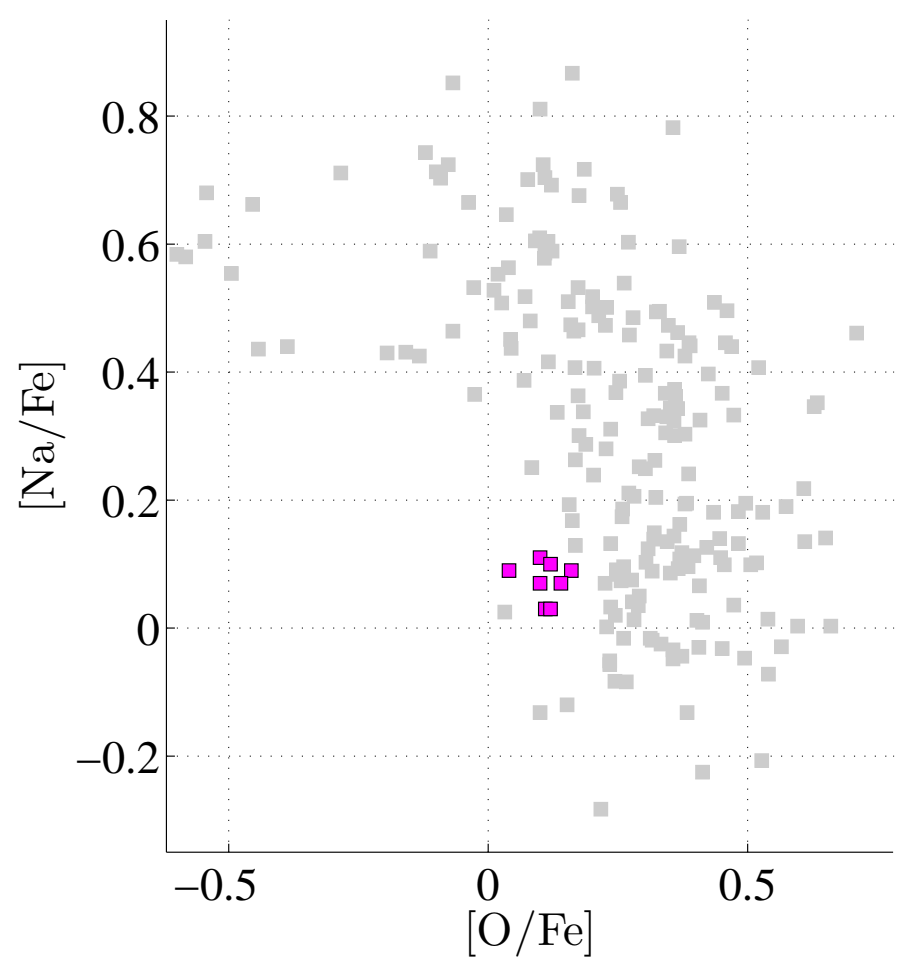

Fig. 2 Na-O diagram of stars in NGC 1806 (pink) and Galactic GCs (gray). The NGC 1806 data were obtained from Mucciarelli et al. (2014); GC data are from Carretta et al. (2009).

\subsection{Stellar Populations in Globular Clusters}

Old GCs appear to represent a separate population of star clusters compared with OCs, YMCs, and also intermediate-age star clusters. Multiple stellar populations are thought to be a common feature of GCs in the Milky Way (Gratton et al.2012). The first evidence of multiple stellar populations in GCs may date back to the 1980s: Hesser \& Bell (1980) found a dispersion in the CN-band strengths 
among seven MS stars in the GC 47 Tucanae (47 Tuc), indicating that a process leading to $\mathrm{N}$ enhancement must have occurred in some of those stars. Since MS stars in 47 Tuc lack the capacity of mixing $\mathrm{N}$ to the stellar surface, such a $\mathrm{CN}$ variation must have a primordial origin. Similar results were also confirmed in other GCs, e.g., in NGC 362 (Smith 1983), NGC 6171 (Smith 1988), M71 (NGC 6838; Penny et al.1992), M3 (NGC 5272; Smith 2002), and M13 (NGC 6205; Smith \& Briley 2006)). N-enhanced stellar populations exhibit a strong absorption line at $\sim 3400 \AA$ : see (Milone et al. 2012, their Fig. 11) and (Piotto et al. 2015, their Fig. 1). Variations of the $\mathrm{N}$ abundance in stellar populations will affect their CMD morphologies in ultraviolet filters, producing a split in the RGB. Such a feature has been detected in the CMDs of a dozen GCs (Piotto et al.2015).

Other evidence of multiple stellar populations in GCs comes from the variations in light elements ( $\mathrm{Na}, \mathrm{O}, \mathrm{Mg}, \mathrm{Al}$ ), including the $\mathrm{Na}-\mathrm{O}$ anticorrelation: see Fig. 2 For more details on light-element chemistry in GCs, we recommend the review by Gratton et al. (2012). The $\mathrm{Na}-\mathrm{O}$ anticorrelation in GCs is exhibited by MSTO, SGB, and RGB stars (Carretta et al. 2004b; Gratton et al. 2004). It has been suggested to result from the CNO cycle $\left(\sim 20 \times 10^{6} \mathrm{~K}\right)$ and the Na proton-capture process $\left(\sim 35 \times 10^{6} \mathrm{~K}\right)$. The central temperatures of the MSTO stars in GCs $\left(\sim 0.85 M_{\odot}\right)$ do not reach the threshold temperature for initiation of the proton-capture process required for the production of $\mathrm{Na}$ (Decressin et al. 2007). Therefore, the $\mathrm{Na}-\mathrm{O}$ anticorrelation is unlikely the result of stellar evolution (e.g., processes affected by the first dredge-up or meridional mixing; Sweigart \& Mengel 1979), but it must instead have a primordial origin intrinsic to the nature of multiple stellar populations. A similar Mg-Al anticorrelation has also been found in GCs (e.g., Gratton et al. 2001; Yong et al. 2003); for more details, see Gratton et al. (2004, 2012). It seems that all Galactic GCs harbor $\mathrm{Na}-\mathrm{O}$ anticorrelations, while light-element variations are also present in some LMC GCs (Johnson et al. 2006).

The iron abundance in a star cluster can only be enhanced through the ejecta of massive, corecollapse supernovae (Cayrel 1986; Parmentier et al. 1999). If a cluster exhibits an iron dispersion, this indicates that its SFH has lived through supernova explosions. Such clusters have been observed: Marino et al. (2009) explored 17 giant stars in the GC M22 and identified two groups with different iron and s-process abundances. A similar result, for M2, was obtained by Yong et al. (2014), who determined a metallicity dispersion exhibiting three peaks, at $[\mathrm{Fe} / \mathrm{H}] \sim-1.7,-1.5$, and -1.0 dex. Such a metallicity dispersion has also been found in the GC NGC 1851 (Carretta et al. 2010). The examples of M2, M22, and NGC 1851 strongly suggest that at least some GCs have experienced much more complex SFHs than provided by individual starburst episodes.

More impressive evidence comes from precise photometry: the multiple stellar groups that show different properties in the CMD have also been found to possess different chemical compositions. For example, NGC 1851 was found to harbor dual SGBs; its two branches were also found to have different CNO contents (Cassisi et al. 2008). D'Antona et al. (2005) studied the color distribution of MS stars in the GC NGC 2808 based on Hubble Space Telescope (HST)/Wide Field Planetary Camera-2 (WFPC2) observations. They found that the width of its MS is inconsistent with an SSP, which requires a helium abundance ranging from $Y=0.26$ to $Y=0.40$. This has been confirmed by Piotto et al. (2007), who obtained accurate photometry of NGC 2808 based on observations with the HST/Advanced Camera for Surveys (ACS). They found that the broadened MS is actually composed of three distinct subsequences, indicating three stellar populations with helium-abundance peaks at $Y=0.248, Y=0.30$, and $Y=0.37$. Spectroscopic evidence was provided by Marino et al. (2014), who analyzed $96 \mathrm{HB}$ stars in NGC 2808 and found that their Na content depends on their position in the CMD, with blue HB stars having higher $\mathrm{Na}$ abundances than their red counterparts. The multiple features in GCs are more apparent in ultraviolet observations (Piotto et al. 2015). A recent study of NGC 7089 (M2) even revealed seven stellar populations (Milone et al. 2015b).

Photometric analyses also enable one to investigate the kinematics and dynamics of the different stellar populations. Milone et al. (2012) found dualities in the MS, SGB, and RGB of 47 Tuc, which can be explained by a $\mathrm{CN}$-weak, O-rich, and Na-poor first stellar generation with a normal 
helium abundance $(Y \sim 0.25)$ and a secondary stellar generation with $\mathrm{CN}$-strong, O-poor, Na-rich elemental abundances as well as an enhanced helium abundance $(Y \sim 0.265)$. The radial behaviors of both stellar generations are different: the second stellar generation is more concentrated than the first generation. Li et al. (2014c) placed their results on a firmer footing through near-infrared observations. They determined more obvious radial stellar population gradients, concluding that the central region of 47 Tuc is almost entirely dominated by the secondary stellar generation, while the cluster's periphery is fully composed of first-generation stars. They also found that the secondary stellar generation has enhanced helium abundance and metallicity. Similar results were also found for NGC 362 (Carretta et al. 2013) and several other GCs (Lardo et al. 2011).

The observational status of stellar populations in star clusters can be summarized as follows:

(a) Despite small numbers of stellar members, extremely young, massive clusters still embedded in their GMCs (usually with ages $\leq 3 \mathrm{Myr}$ ) are characterized by proto-stellar populations that formed in a single episode with a very small age spread.

(b) Intermediate-age LMC and SMC star clusters (but also including NGC 1850 and NGC 1856, which have ages of only $150 \mathrm{Myr}$ and $300 \mathrm{Myr}$, respectively) exhibit eMSTO regions. If their extents are interpreted as fully caused by a range in stellar ages, they are in apparent conflict with the SSP scenario. However, no evidence of chemical dispersions has been found in these clusters (Mucciarelli et al. 2014). The tight SGBs in some of these clusters also contradicts a significant age spread (Li et al. 2014b, 2016a, Bastian \& Niederhofer 2015). Whether these clusters may be characterized by initially extended SFHs is still being debated.

(c) The presence of multiple stellar populations in GCs is undisputed, making it the greatest open challenge to the SSP scenario for star clusters. A combination of spectral and photometric evidence supports the presence of multiple stellar populations in GCs, which also differ in both their kinematics and dynamics. This may indicate primordial chemical inhomogeneities. However, as we will show in Section 4, stellar evolution is probably still a straightforward solution to explain the observations of some of these clusters.

\section{PROPOSED SCENARIOS}

\subsection{Controversy about Multiple Stellar Populations in Young and Intermediate-age Clusters}

An intuitive explanation of the eMSTO regions observed in intermediate-age star clusters is that their member stars may have formed during a time span of several hundred million years. Since the morphology of the MSTO is very sensitive to the age distribution of its stellar population, many authors have suggested that eMSTO regions in intermediate-age clusters might imply eSFHs of at least 300 Myr. Goudfrooij et al. (2014) proposed a model for massive clusters characterized by a prolonged round of star formation: the central escape velocities of their eMSTO model star clusters were sufficiently high to accrete a significant amount of pristine gas from their surroundings or to retain the gas from the ejecta of intermediate-mass AGB stars and massive binaries. Since the remnant gas does not fully escape from the infant clusters, most of this gas reservoir would be accumulated in the central regions of the clusters, subsequently forming secondary populations of stars. This process would last several million years until all gas is exhausted by star formation or multiple supernova explosions. However, the lack of residual gas in YMCs (Bastian et al. 2013) all but invalidates this scenario. A second proposed origin of such an age spread is the merger between two clusters with different ages (Mackey \& Broby Nielsen 2007) or between a cluster and a starforming GMC (Bekki \& Mackey 2009). If this scenario turns out to work, it would imply that most LMC clusters actually belonged to binary systems in the past, since $70 \%$ of intermediate-age star clusters in the LMC show such eMSTOs (Milone et al. 2009). However, presently binary clusters only account for about $10 \%$ of the current cluster population of the Magellanic Clouds (Dieball \& Grebel 2000). 
An alternative scenario to explain eMSTOs draws on a range in stellar rotation rates. Stellar rotation can alter the morphology of MSTO regions in two ways: (i) the centrifugal force resulting from rotation reduces the stellar self-gravity, thus decreasing the surface effective temperature, which in turn renders its observed color redder than that of the non-rotating counterparts. The reduction in stellar self-gravity also decreases the stellar core pressure, hence reducing the central nuclear reaction rate (Faulkner et al. 1968), which causes a dimming of fast rotators compared with slowly or non-rotating stars. The observed stellar luminosities and colors also depend on the inclination angles of individual stars: a rapidly rotating star will look redder around its equatorial region than near its poles. This effect is known as 'gravity darkening' (see Georgy et al. 2014, their Fig. 1). (ii) Rotation enlarges the stellar convective cores, transporting hydrogen from the outer layers into the central region, in turn replenishing the central fuel for hydrogen burning, which may thus prolong the stellar MS lifetime. This is called 'rotational mixing.' It has been suggested that stellar rotation of O-, early-B-, and F-type stars may lead to lifetime increases by about 25\% (Maeder \& Meynet 2000; Girardi et al. 2011).

Less massive stars $\left(\leq 1.2 M_{\odot}\right)$ are not expected to become fast rotators because of magnetic braking (Schatzman 1962, Mestel \& Spruit 1987), i.e., the stellar magnetic field exerts a torque on the ejected matter during stellar evolution, resulting in a steady transfer of angular momentum away from the star. For example, stars earlier than F0-type can easily reach an average rotational velocity of $100-200 \mathrm{~km} \mathrm{~s}^{-1}$, while the typical rotational velocity of G0-type stars is only $12 \mathrm{~km} \mathrm{~s}^{-1}$ (McNally 1965). A more recent study of a large number of B-F-type solar-neighborhood stars was carried out by Royer et al. (2007), who confirmed that most of these stars are fast rotators.

Bastian \& de Mink (2009) suggested that rapid stellar rotation of F-type stars may lead to the misconception that intermediate-age star clusters harbor significant age spreads. In their scenario, the dominant effect that is responsible for the extent of the (e)MSTO region is gravity darkening. However, Girardi et al. (2011) countered that their model is unrealistic, because it ignores rotational mixing. The latter authors showed that rotational mixing will mitigate the broadening caused by gravity darkening. As a result, the combination of these two effects may still produce a narrow MSTO, and therefore, they argued, stellar rotation cannot be the (only) cause of the eMSTO. However, Yang et al. (2013) pointed out that the conclusion of Girardi et al. (2011) is tenable only in the presence of a high convective mixing efficiency. Yang et al. (2013) studied the collective effects of gravity darkening and rotational mixing with a moderate mixing efficiency. They successfully reproduced the eMSTO regions for 1-2 Gyr-old clusters. A corollary of their results is that the MSTO area in a cluster depends on its actual age (see also Brandt \& Huang 2015). Niederhofer et al. (2016) found a correlation between the full width at half maximum (FWHM) of the postulated age spreads in clusters and their actual ages (based on isochrone fitting to the blue boundary of their eMSTO regions) for 12 intermediate-age LMC star clusters, which is consistent with the predictions of the stellar rotation scenario. In Fig. 3 we present the predicted correlation between the FWHM of the clusters' age spreads and their typical ages for coeval stellar populations (reproduced from Fig. $8 \mathrm{f}$ of Yang et al.|2013); observational data are also included (Goudfrooij et al. 2014; Correnti et al. 2015, Niederhofer et al. 2015b).

Figure 3 shows that the MSTO regions of clusters with ages of up to $2.5 \mathrm{Gyr}$ are more extended than expected for SSPs. The maximum age spread derived from the eMSTO region occurs for ages ranging from $\sim 1.5$ Gyr to 2.0 Gyr. In Yang et al. (2013)'s model, a positive FWHM means that gravity darkening is the dominant effect, making the fast rotators redder than their non- or slowly rotating counterparts. On the other hand, a negative FWHM implies that rotational mixing dominates, resulting in fast rotators to appear bluer than the non- or slow rotators. Brandt \& Huang (2015) also found that the area of a cluster's MSTO region depends on its age if its MSTO stars have different rotation rates. However, their models imply that rotational mixing is likely the dominant effect responsible for the extent of the eMSTO region at any age, i.e., the fast rotators are bluer 


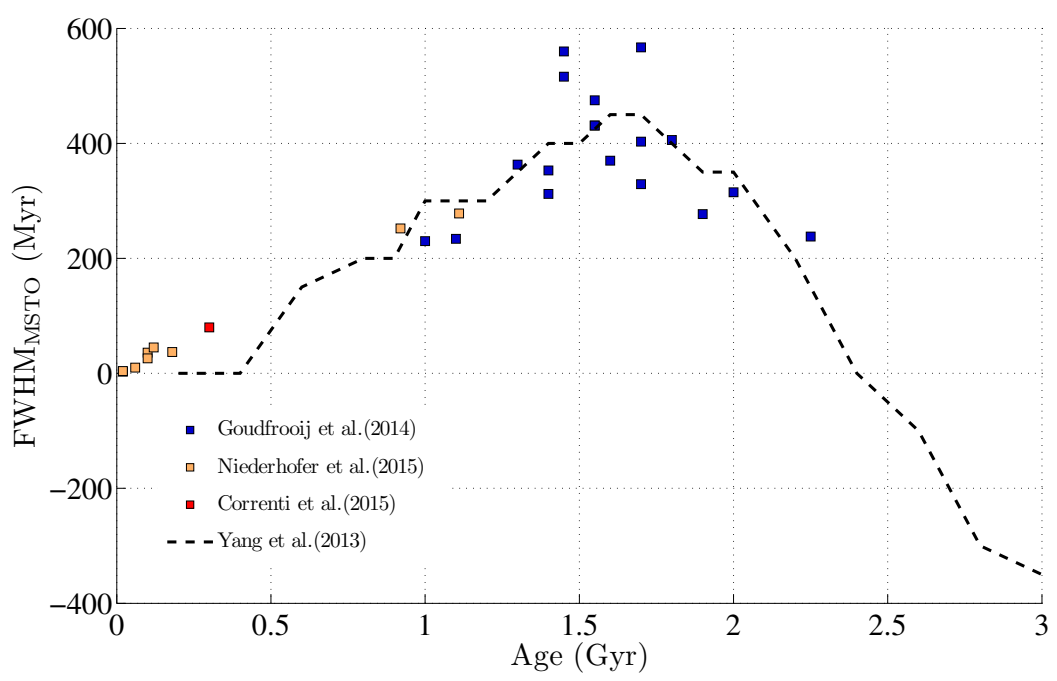

Fig. 3 Widths of implied cluster age spreads as a function of isochronal age. Black dashed line: Predicted FWHM of cluster age spreads that would be derived from their eMSTO regions as a function of cluster age. A positive (negative) FWHM means that the populations of fast rotators are redder (bluer) than the non- or slowly rotating populations. Blue, red, and orange rectangles: star cluster data from Goudfrooij et al. (2014), Niederhofer et al. (2015b), and Correnti et al. (2015), respectively.

than the non- or slow rotators, predominantly owing to rotational mixing. The maximum age spread derived from their MSTO regions peaks at $t \in[1.0,1.5] \mathrm{Gyr}$.

If the apparently significant age spreads of several hundred million years are indeed valid for YMCs $(\leq 300 \mathrm{Myr})$, one should expect pre-MS stars to appear in YMC CMDs, i.e., the age distributions of their member stars would scatter to zero age. However, as shown in Fig. 3, the internal age spreads of YMCs derived from their eMSTO regions only span small fractions of their ages. Once again, this supports the rapid stellar rotation scenario, since it will only partially broaden the MSTO region.

The relative importance of gravity darkening and rotational mixing in star clusters is as yet unclear owing to a lack of direct observational evidence. The main difference between these processes resides in the loci of the rapidly rotating population. Gravity darkening will produce an eMSTO where most fast rotators reside toward the red side of the MSTO, while rotational mixing will result in fast rotators being distributed on the blue side of the MSTO region. However, measuring stellar rotation in dense star clusters at the distance of the LMC is difficult with current facilities. Since both stellar rotation and age spreads will produce eMSTOs in star clusters, important differences which may allow us to distinguish between these processes may appear on the SGB. Because gravity darkening does not produce a mass spread among MSTO stars, once the MSTO stars have evolved off the MS, they will slow down rapidly as a result of the conservation of angular momentum. Subsequently, the coeval MSTO population characterized by different stellar rotation rates will converge into a narrow SGB (Wu et al. 2016). However, rotational mixing will produce a mass spread among evolved stars, which will still broaden both the MSTO and SGB regions. In Table 1 we summarize the features of the MSTO region and the SGB for four cases: (1) an SSP without stellar rotation; (2) a 
Table 1 MSTO and SGB features corresponding to (1) an SSP, (2) an age spread (eSFH), (3) gravity darkening, and (4) rotational mixing.

\begin{tabular}{lcc}
\hline Scenario & MSTO Region & SGB Region \\
\hline SSP & Narrow & Narrow \\
eSFH & Extended in color and luminosity & Extended in luminosity \\
Gravity darkening & Extended in color and luminosity & Narrow \\
Rotational mixing & Extended in color and luminosity & Extended in luminosity \\
\hline
\end{tabular}

significant age spread (eSFH); (3) an SSP with fast rotators affected by gravity darkening; and (4) an SSP with fast rotators affected by rotational mixing.

The Geneva stellar evolutionary code includes the effects of initial stellar rotation. 1 Ekström et al. 2012, Georgy et al. 2013a b; Yusof et al. 2013) but it currently only covers a limited stellar mass range and a small number of fixed initial rotation rates. (Observationally, we only have access to current stellar rotation rates) ${ }^{2}$ In Fig. 4 we present synthetic CMDs of SSPs with $\Omega_{\text {ini }} / \Omega_{\text {crit }} \leq 0.7$ (left) and $\Omega_{\text {ini }} / \Omega_{\text {crit }} \geq 0.7$ (right) evolved to an age of $1 \mathrm{Gyr}$ and for a metallicity of $Z=0.006$, i.e., the value closest to that typical of most intermediate-age LMC and SMC star clusters. Application of the Geneva code shows that for initial stellar rotation rates of $\Omega_{\text {ini }} / \Omega_{\text {crit }} \leq 0.7$, rotational mixing is the dominant effect responsible for broadening the MSTO region: the relatively rapid rotators are bluer than their slowly rotating counterparts. In contrast, for stars with $\Omega_{\text {ini }} / \Omega_{\text {crit }} \geq 0.7$, the fast rotators are redder than the slow rotators (which also have initial rotation rates $\Omega_{\text {ini }} / \Omega_{\text {crit }} \geq$ 0.7), indicating a dominant importance of gravity darkening among the rotating stellar population. A remarkable difference appears on their SGBs, since rotational mixing produces a mass spread for evolved stars, which thus causes a broadened SGB. However, as shown in the right-hand panel of Fig. 4, gravity darkening will not broaden the SGB.

A breakthrough was achieved by Li et al. (2014b) and Bastian \& Niederhofer (2015). Both independent groups found that the SGB morphologies in three star clusters, NGC 1651, NGC 1805, and NGC 1846, were inconsistent with significant age spreads. However, their conclusions were subsequently challenged by Goudfrooij et al. (2015), who argued that if one were to consider the effects of stellar convective overshooting, the morphologies of the clusters' SGBs may still be consistent with the presence of significant age spreads. The debate thus turned to whether overshooting is important for F-type SGB stars. Colucci \& Bernstein (2012) used a sample of young and intermediate-age LMC clusters to evaluate the importance of overshooting. They found that the use of isochrones without convective overshooting results in $\mathrm{Fe}$ abundances that most closely match the loci of stars in clusters with ages between 0.05 Gyr and 3 Gyr. Another striking contrast between the widths of the eMSTO region and the SGB is exhibited by the SMC cluster NGC 411, which hosts an eMSTO region that implies an apparent age spread of $\sim 800 \mathrm{Myr}$, but it harbors a extremely tight SGB, which can only be described by a single-aged isochrone (Li et al.2016a). In Fig. 55 we present the CMDs of (left) the LMC cluster NGC 1651 and (right) the SMC cluster NGC 411. The best-fitting isochrones that cover their eMSTO regions (indicated by the purple outlines) are also shown; the SGB regions are indicated by regions traced by black dashed lines. One can immediately see that, compared with their eMSTO regions, the clusters' SGBs do not show the expected corresponding broadening or bifurcation. This combined analysis of cluster eMSTO regions and tight SGBs indeed disfavors the age-spread scenario. Instead, only an extremely rapidly rotating population will produce an eMSTO combined with a tight SGB (see the comparison in Fig. 4), which may indicate that stellar rotation in star clusters occurs at much higher velocities than previously expected (Wu et al. 2016).

\footnotetext{
1 http://obswww.unige.ch/Recherche/evol/-Database

2 The Geneva models are only available for nine different initial rotation rates, $\Omega_{\text {ini }} / \Omega_{\text {crit }}=0.0,0.1,0.3,0.5,0.6,0.7$, $0.8,0.9$, and 0.95 , where $\Omega_{\mathrm{ini}}$ is the initial stellar angular rotation rate and $\Omega_{\text {crit }}$ is the critical, 'break-up' value. The stellar
} 

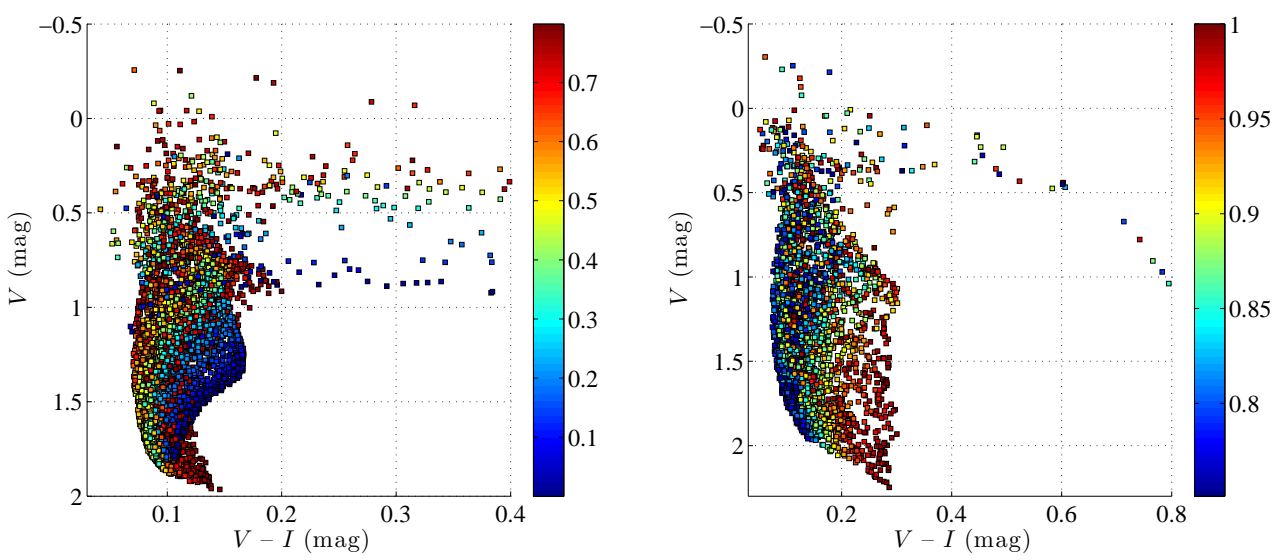

Fig. 4 Synthetic $(V, V-I)$ CMD for a coeval stellar population (based on the Geneva models) evolved to an age of $1 \mathrm{Gyr}$ and with a metallicity of $Z=0.006$, for stars with initial rotation rates of (left) $\Omega_{\text {ini }} / \Omega_{\text {crit }} \leq 0.7$ and (right) $\Omega_{\text {ini }} / \Omega_{\text {crit }} \geq 0.7$. The color bars represent the initial stellar rotation rates.

masses included range from $1.7 M_{\odot}$ to $15 M_{\odot}$. In addition, for the mass range between $0.8 M_{\odot}$ and $120 M_{\odot}$, the model suite is defined for two initial rotation rates, $\Omega_{\text {ini }} / \Omega_{\text {crit }}=0.0$ and 0.568 .
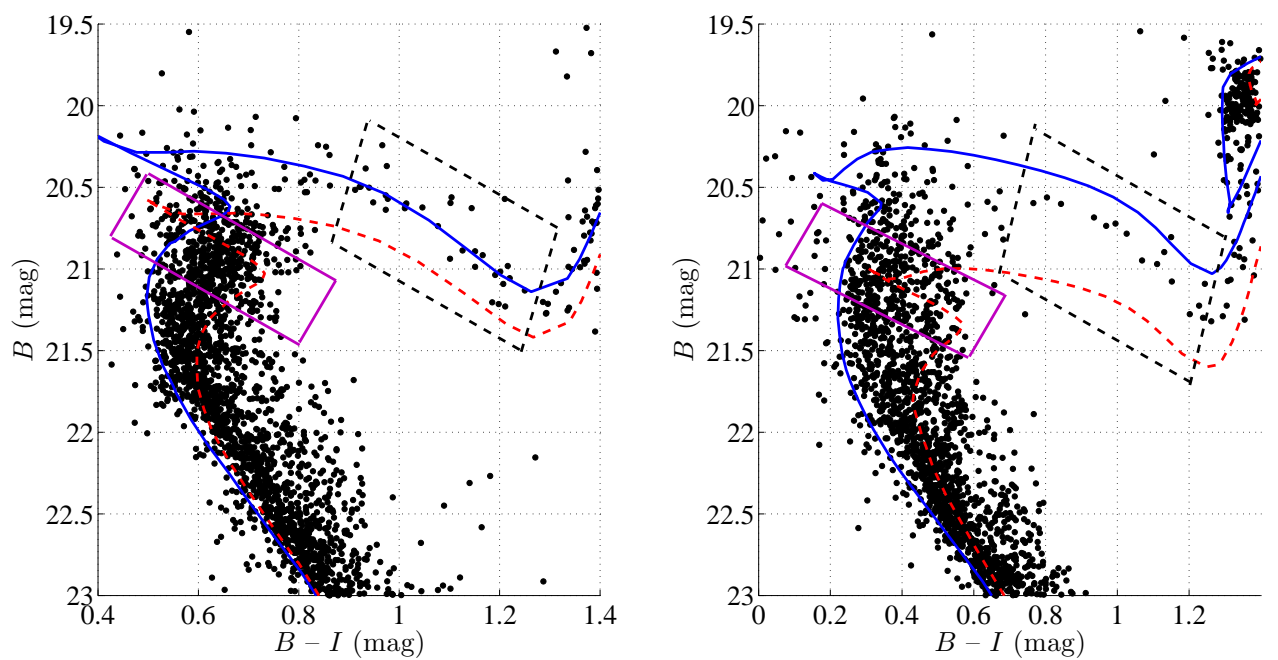

Fig. 5 CMDs of the intermediate-age star clusters NGC 1651 (left) and NGC 411 (right). Both harbor eMSTO regions (purple outlines) and well-populated SGBs (black dashed outlines). The best-fitting isochrones (blue, red solid lines: young, old isochrones) are also shown, determined based on the extents of their eMSTO regions. 
The discovery of eMSTOs in YMCs further confirms this. The eMSTO of NGC 1856, a 150 Myr-old YMC, suggests an $\sim 80$ Myr age spread (Correnti et al. 2015). Correnti et al. (2015) concluded that if the observed eMSTO is caused by stellar rotation instead, then rotational mixing will reproduce the observations if the stellar population is composed of a combination of two-thirds rapidly rotating stars and one-third slowly/non-rotating stars. The same conclusion was reached by Milone et al. (2016), who discovered a split main sequence in the YMC NGC 1755. This feature favors the variable stellar rotation scenario rather than an age spread. They considered whether all stars in NGC 1755 were born rapidly rotating. All these studies were based on the premise that rotational mixing is the dominant effect in determining the extent of an eMSTO. If, on the other hand, stellar rotation were responsible for the eMSTOs of YMCs, rotational mixing should be of significant importance. Thus, to further constrain the internal age distributions and the relative importance of rotational effects, future investigations of the SGBs or RCs of intermediate-age star clusters are urgently required.

In summary, the apparent eMSTO regions of intermediate-age star clusters and YMCs in the LMC and SMC can be explained by scenarios postulating either an age spread or rapid stellar rotation. The field still has a long way to go before reaching a unanimous conclusion.

\subsection{Possible Origins for Multiple Stellar Populations in Globular Clusters}

Unlike for intermediate-age star clusters, the presence of multiple stellar populations in old GCs is well-established. Scenarios that have been proposed to explain the formation of multiple stellar populations in old GCs are diverse, but most can be grouped into two categories. The first type of hypothesis suggests a primordial origin, in the sense that GCs may have been born with chemical inhomogeneities, i.e., GCs were formed with a primordial chemical dispersion. The second type of scenario favors the SSP origin, and the multiple stellar populations in GCs are then the result of stellar evolution (see also Kraft 1994).

Most primordial scenarios draw on self-pollution of intra-cluster gas, occurring during the early stages of cluster evolution. Various polluters have been proposed, including those originating from the ejecta of rapidly rotating massive stars (Decressin et al. 2007), massive binaries (de Mink et al. 2009), or evolved post-giant-branch stars (Ventura \& D'Antona 2009). Valcarce \& Catelan (2011) proposed a scenario that divides GCs into three groups, where the most massive GCs, with initial masses $\geq 10^{9} M_{\odot}$ (e.g., M22), can retain the ejecta of all types of massive stars, including those of their core-collapse supernovae. Intermediate-mass GCs, with masses of several $\times 10^{8} M_{\odot}$ (like NGC 2808) would only be able to retain a fraction of the fast winds from massive stars. The least massive GCs would not be able to retain any gas ejected by massive stars (or their products), but they could form new stars from the slow winds of intermediate-mass stars of the first stellar generation. Self-enrichment scenarios usually postulate multiple episodes of star formation. If confirmed, this means that we may have underestimated the capacity of GCs to collect and retain gas. Recently, D'Antona et al. (2016) proposed a similar scenario. They claimed that a temporal sequence of AGB generations may be responsible for the observed multiple stellar populations in GCs: the pollution process occurs after the Type II supernovae epoch, lasting until the third dredge-up associated with the AGB population. They ascribe the cluster-to-cluster abundance variations to differences in many processes and gas sources which were involved in the formation of the secondary generation. Based on this scenario, after the Type II supernovae epoch ( $20 \mathrm{Myr})$, GCs should still have been 100 times more massive then their current masses; otherwise the self-pollution scenario would be hard pressed to explain the high fraction of secondary stellar generations observed.

Bastian et al. (2015) carefully studied all current self-enrichment scenarios by comparing them to observations. They found that an intrinsic problem among all these scenarios is that they are unable to produce consistent abundance trends among $\mathrm{He}, \mathrm{Na}$, and $\mathrm{O}$. To form a secondary stellar generation, clusters should be able to retain or accrete material from their surroundings (Conroy \& 
Spergel 2011), but current investigations have shown that clusters are almost gas-free at a very early stage (2-3 Myr), independent of their mass (Bastian et al. 2014; Hollyhead et al. 2015). While these results suggest that clusters may not be able to retain their gas during the initial gas-expulsion phase, this scenario does not prevent a cluster from accreting external gas onto its core potential while moving through a background medium (Naiman et al. 2011).

An unexpected discovery that relatively young, 1-2 Gyr-old star clusters can harbor secondary stellar populations was made by Li et al. (2016b), who found that the LMC clusters NGC 1783 and NGC 1806, as well as the SMC cluster NGC 411, host secondary stellar populations that are genuinely younger than their first-generation stars by about 500 Myr to 1 Gyr. The observed, different young stellar populations are tightly associated with younger isochrones, indicating that they are strictly coeval. However, these young stellar populations are somewhat less centrally concentrated compared with the first-generation RGB stars in the same clusters. These authors speculated that the younger stars may have an external origin, which renders the gas-accretion scenario promising once again. However, because the observed young stellar populations in those extragalactic star clusters only occupy minor mass fractions, additional numerical simulations are required to further study the implications of these observations. In Fig. 6 we present the observed CMD of NGC 1783 as an example. Although the young stars have a slightly more extended nature compared to the approximately equal-luminosity RGB stars, they are firmly concentrated within 1 to 2 core radii. The observed young stellar population stars have a number-density profile that clearly peaks in the clusters' core regions. This cannot be explained by invoking background contamination (see Fig. 7), which implies that these newly born stellar populations indeed physically belong to their host star clusters.

Another primordial formation scenario is based on cluster mergers, which is the prevailing model for some of the most massive GCs, such as NGC 1851 (Carretta et al.2010). An advantage of this hierarchical formation scenario is that it solves the 'initial exhaustion' problem. This scenario preferentially favors clusters that are located in crowded environments, such as the so-called 'clusters of clusters' (e.g., cluster pairs in the Antennae interacting system; Bastian et al.2009). Cluster members in these environments are expected to frequently collide and merge (Amaro-Seoane et al.|2013). However, most GCs in our Milky Way are located in the Galactic halo, where frequent mergers are not expected to occur.

The evolutionary scenario attributes the observed abundance variations to the dredge-up of material that has been processed through the CNO cycle in the cluster stars themselves. Because the observed chemical dispersions of different elements in GC stars only represent variations in the stellar surface abundances, any process that can somehow transfer core material to the stellar surface would produce star-by-star variations in elemental abundances. Because the evolutionary scenario strongly depends on the efficiency of stellar convective mixing, various mechanisms that may affect the mixing of stellar material have been proposed. The most promising scenario suggests that stars will undergo deep mixing when they evolve off the MS. Lee (2010) carefully studied the $\mathrm{Na}-\mathrm{O}$ distributions in Galactic GCs. They found a dependence of the Na-O anticorrelation on RGB luminosity. This is probably owing to the internal deep mixing of evolved stars during their ascent of the RGB. Their results thus partially support the evolutionary scenario.

Another possible mechanism considers stellar magnetic fields: Nucci \& Busso (2014) proposed a scenario involving the advection of thermonuclear ashes by magnetized domains emerging near the $\mathrm{H}$ shell to explain AGB-star abundances. Based on magnetohydrodynamics calculations, they verified that the stellar-envelope crossing times are sufficient to facilitate chemical dispersion in a huge convective shell. They claimed that magnetic advection is a promising mechanism for deep mixing, which may explain the observed abundance anomalies in GCs. However, because stars in GCs are usually late-G- or K-type stars, the central temperatures of individual low-mass stars do not reach the threshold for producing the observed $\mathrm{Na}-\mathrm{O}$ anticorrelation. Jiang et al. (2014) proposed a model based on fast rotators that are produced by binary mergers or interactions. They found for binary merger products which are normal, rapidly rotating stars earlier than F-type, that these rapidly 


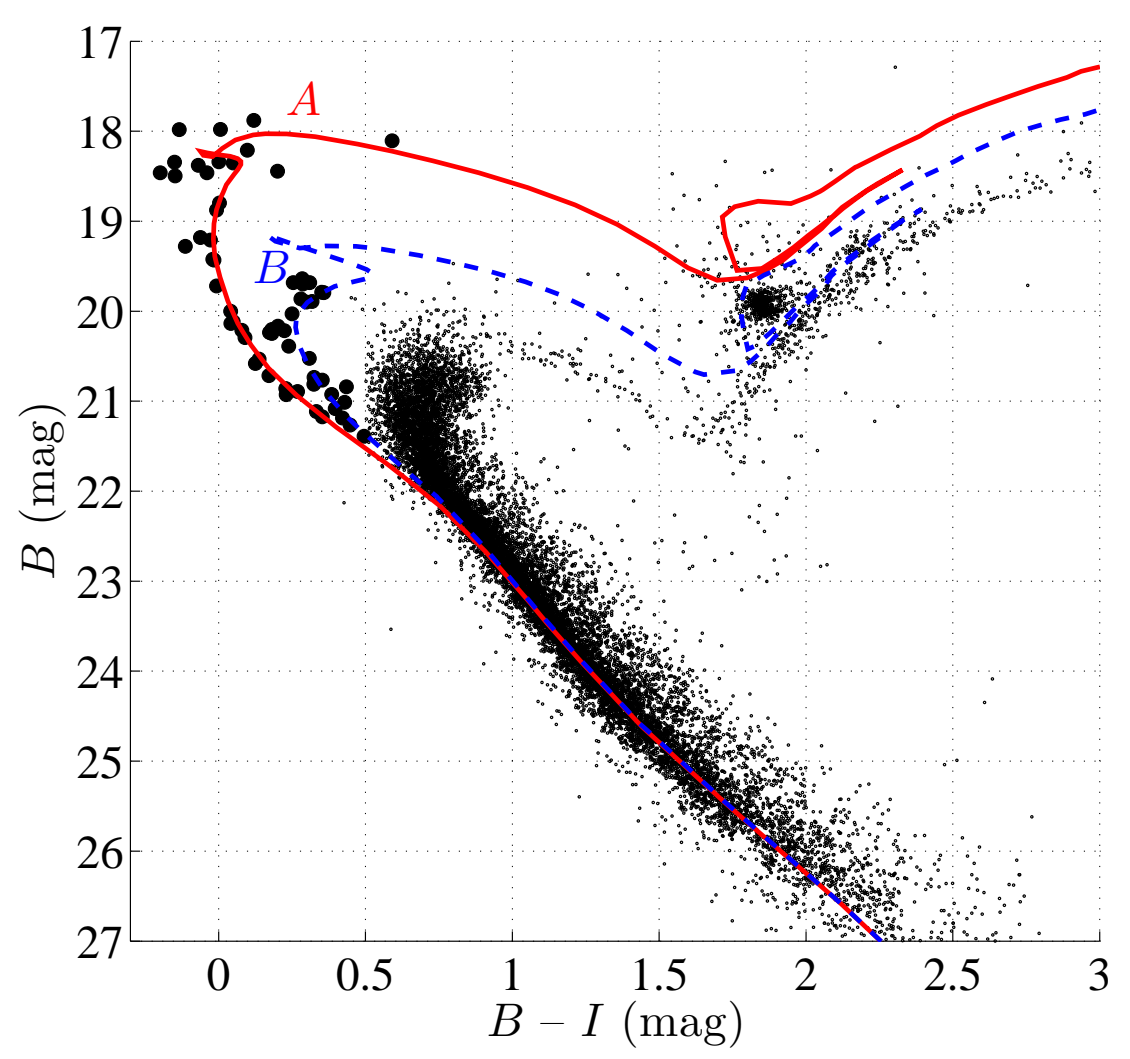

Fig. 6 CMD of the LMC cluster NGC 1783 (1.4 Gyr old). NGC 1783 hosts two apparently younger stellar populations, which are tightly associated with two isochrones (shown as the blue dashed and the red solid lines), indicated as populations A and B. Similar features are also found in the LMC cluster NGC 1806 and the SMC cluster NGC 411.

rotating stars can produce a $\mathrm{Na}-\mathrm{O}$ anticorrelation with high significance. They claimed that binary interactions are a possible solution to the chemical anomalies in GCs.

The evolutionary scenarios do not require an extremely massive origin for clusters to generate abundance anomalies. Jiang et al. (2014)'s scenario only depends on a cluster's binary fraction. This implies that OCs would also display $\mathrm{Na}-\mathrm{O}$ anticorrelations if they are sufficiently old for large numbers of their binaries to have merged. However, so far we do not have any conclusive evidence that OCs may harbor multiple stellar populations. Cantat-Gaudin et al. (2014) studied the chemical homogeneity of RC stars in the OC NGC 6751, but no obvious correlations or anticorrelations among $\mathrm{Al}, \mathrm{Mg}, \mathrm{Si}$, and $\mathrm{Na}$ were found. A similar conclusion was also reached by Bragaglia et al. (2014), who studied 35 evolved stars in an old OC, NGC 6791 (8-9 Gyr old; Brogaard et al. 2012). They did not detect any significant star-to-star chemical dispersions in C, N, O, or Na either. In Fig. 8 we present the performance of the scenario of Jiang et al. (2014). In the top panel, we show the theoretical CMD of an SSP following binary mergers; in the bottom panel, we show the $\mathrm{Na}-\mathrm{O}$ anticorrelation produced by this model compared with observations made in GCs. 


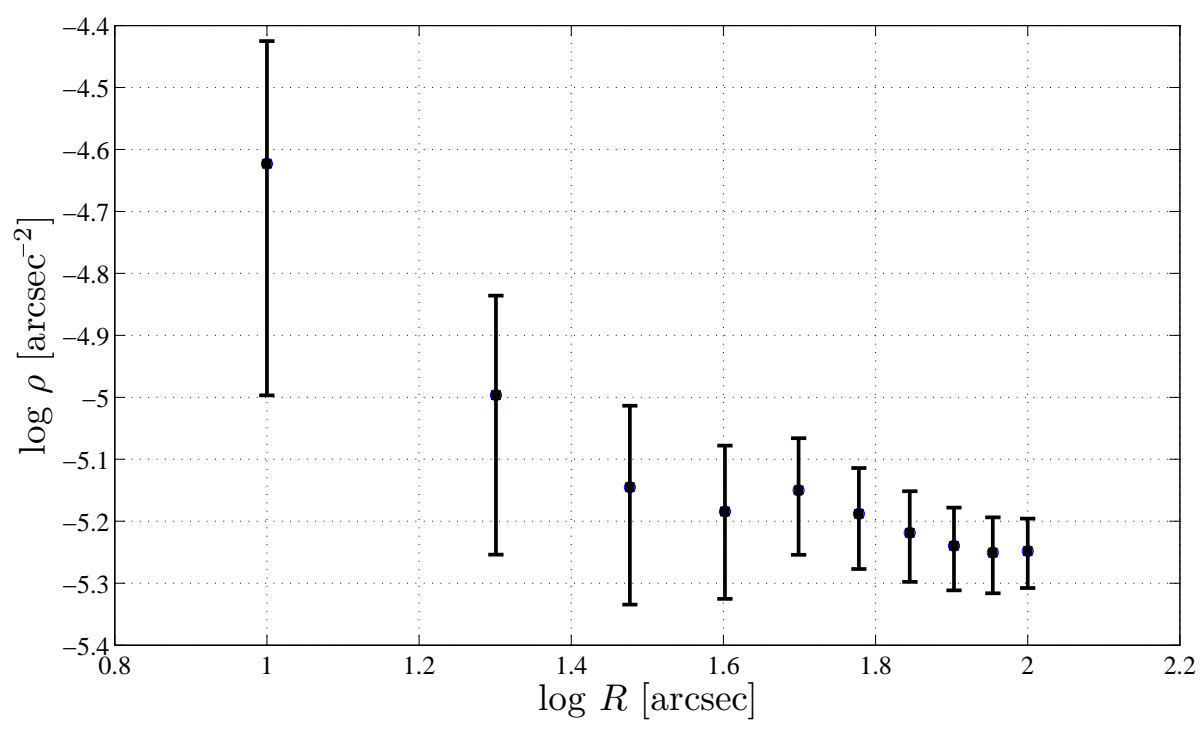

Fig. 7 Number-density profile of young stellar population stars in NGC 1783 (see also the CMD in Fig. 6).

Figure 8 shows that there is a clear correlation between the stellar $\mathrm{Na}$ (as well as the $\mathrm{O}$ ) abundance and luminosity. The binary products are, in fact, 'blue straggler stars' (BSSs). For more details about the primordial and evolutionary scenarios for the origin of multiple stellar populations in GCs, we recommend the review by Kraft (1994); see also Denissenkov et al. (2015).

\section{PROSPECTS}

\subsection{Studying Stellar Populations in Supermassive Star Cluster Candidates using Next-Generation Telescopes}

The origin of multiple stellar populations in star clusters is a subject of significant current debate. To reach closure on this question, the most straightforward approach would consist of observing a 'young GC' and resolving its initial stellar population(s). Most scenarios suggest that a cluster's mass plays a key role in the existence of multiple populations. The most popular scenarios suggest that GCs should initially have been 10 to 100 times more massive than their current masses. Therefore, a 'good' candidate for follow-up observations should be a young cluster with a mass of at least $10^{6}$ $M_{\odot}$. To date, no such supermassive young clusters have been observed in either the Milky Way or its satellites.

Strictly speaking, young GCs should reside at a redshift of $z \sim 2$, which is even beyond the ability of the James Webb Space Telescope to resolve. Therefore, resolving such young clusters will not be feasible in the near future. However, some candidate extremely massive young GCs have already been detected in nearby starburst galaxies. Smith et al. (2006) found five possible supermassive cluster candidates in the galaxy M82. The mass of one of these 'clusters,' M82-A1, is $1.3_{-0.4}^{+0.5} \times 10^{6} M_{\odot}$, at an age of only $6.4 \pm 0.5 \mathrm{Myr}$. If this object truly is an individual YMC, it may be the best candidate GC predecessor known. However, our current facilities are not yet able 

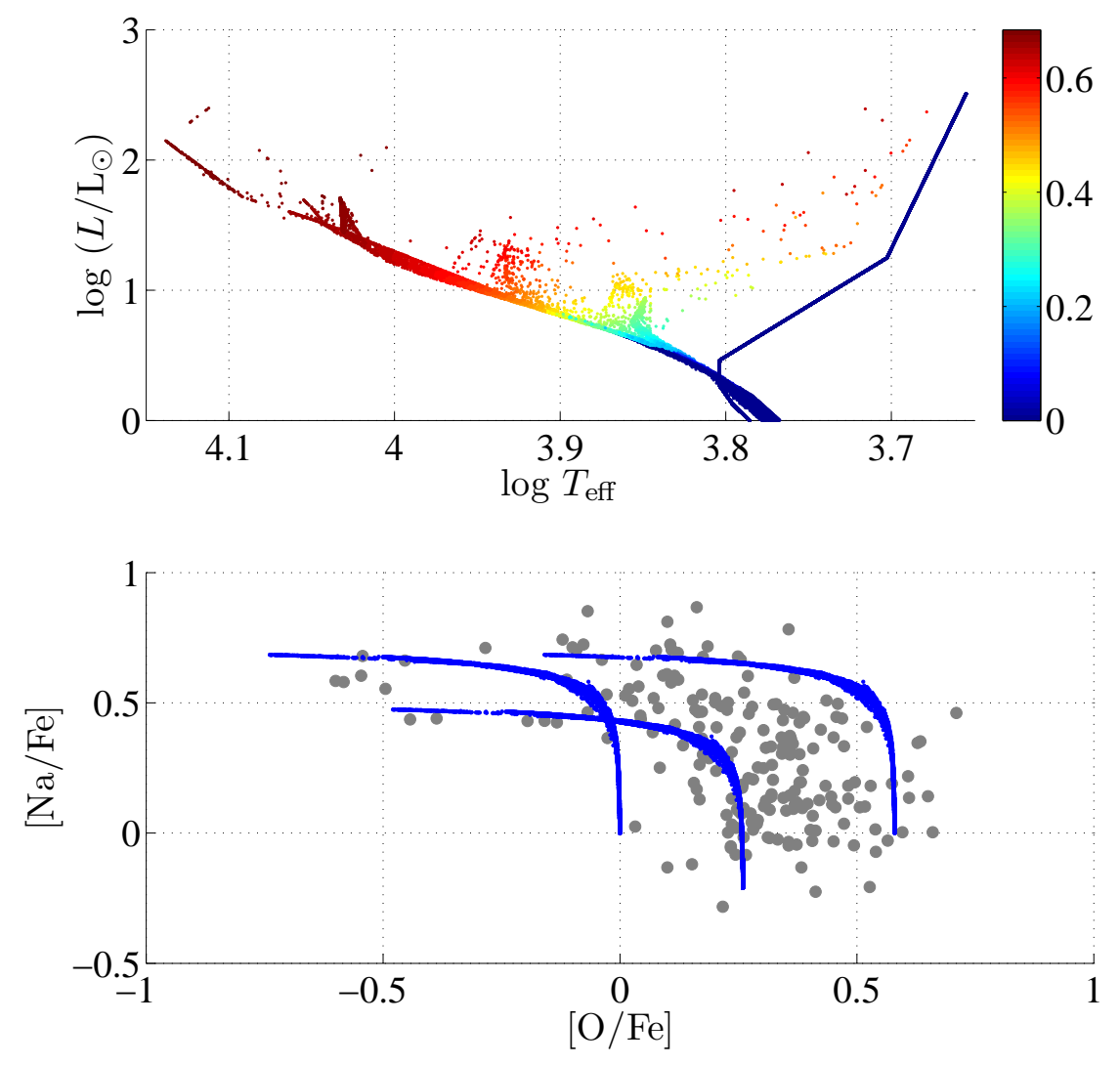

Fig. 8 The importance of binary systems in realistic stellar populations. Top: CMD of an SSP including binary interactions. Color bar: stellar Na abundance. Bottom: Na-O relationships produced by the model (blue) compared with observations (gray bullets). Each sequence of blue dots represents an SSP, characterized by different initial abundances.

to resolve individual stars in such supermassive young star clusters, and only synthetic spectra are available to study their stellar populations (e.g., Cabrera-Ziri et al.2015).

Once next-generation telescopes have been developed, a top priority to improve our understanding in this field will be to resolve individual stars in these candidate young GCs. If these supermassive young objects are confirmed as individual star clusters (and not blended pairs or groups of clusters), then studying their stellar populations will significantly help us constrain the origin of GCs. Using the CMDs of these supermassive young star clusters, we can determine their ages and any internal age dispersions, which is of fundamental importance for studying the star-formation mode in young GCs. If the multiple stellar populations observed in GCs represent multiple episodes of star formation, then one should detect dispersed age distributions in all supermassive young star clusters. This means that large fractions of pre-MS stars are expected to reside in those clusters, and their residual gas should still represent a large mass fraction. 


\subsection{Measuring Stellar Rotation Rates in Intermediate-age Star Clusters}

The 'eMSTO problem' associated with intermediate-age star clusters has shown that rapid stellar rotation is an important process that may confuse our understanding of the SFHs of star clusters. Although measuring stellar rotation rates of individual stars in compact star clusters at the distance of the LMC is challenging, some current instruments can already obtain direct measurements of stellar rotation in LMC star clusters. Using the Multi Unit Spectroscopic Explorer (MUSE) at the European Southern Observatory's Very Large Telescope (VLT), one can in principle obtain lowresolution spectra of all stars resolvable by MUSE in a $1 \operatorname{arcmin}^{2}$ field covering the central regions of intermediate-age LMC star clusters, including of their F-type eMSTO stars. If rotation is the cause of the eMSTO, one will immediately obtain a clear and unequivocal signature in support of this.

One can quantify stellar rotation rates by measuring line broadening in eMSTO stars. The MgI triplet around $5175 \AA$ is an ideal indicator of the stellar rotation rate. Adopting the rapid stellar rotation scenario, a stellar rotation rate from zero to a maximum of $70 \%$ of the break-up rate will be responsible for the observed eMSTO in intermediate-age star clusters. This corresponds to equatorial velocities of $\sim 300 \mathrm{~km} \mathrm{~s}^{-1}$. The MUSE spectral resolution will allow us to resolve differences between no rotation and rotation rates as low as (50-)100 $\mathrm{km} \mathrm{s}^{-1}$, with a signal-to-noise ratio of 30 . In Fig. 9 we present the simulated morphology of the MgI triplet of F2V stars for different rotation velocities, as they would be observed with MUSE. As long as there are no nearby stars in the same resolution element with intensities within $1 \mathrm{mag}$ of the target stars, one will be able to derive a sample of stellar radial velocities, velocity dispersions, and the overall distribution of (eMSTO and other) rotation rates in such clusters. Such a project will provide a definitive answer to the importance of the stellar rotation scenario with respect to the internal age-spread model as regards the origin of eMSTOs in intermediate-age star clusters.

\subsection{Exploring the Relationship between Elemental Abundances and the Luminosities of Blue Straggler Stars}

If the scenario of Jiang et al. (2014) is correct, a correlation between elemental abundances and the luminosities of BSSs is expected. BSSs are rejuvenated MS stars, formed through either stellar collisions (Hills \& Day 1976) or binary mass transfer (McCrea 1964; Carney et al. 2001). The relative importance of these two formation channels during different evolutionary stages in star clusters has not yet been determined unequivocally from an observational perspective. However, numerical simulations have shown that the number of BSSs of binary origin will continue to dominate the total BSS sample over cosmic timescales ( $10 \mathrm{Gyr})$. The number of collisional BSSs becomes comparable to the number of BSSs originating from binary interactions on timescales exceeding a Hubble time (Hypki \& Giersz 2013).

Jiang et al. (2014)'s scenario draws on binary mergers to generate a population of fast rotators, producing surface abundance anomalies through evolution of the latter. A promising approach to examine this scenario is by studying the correlation between luminosity and elemental abundances as pertaining to BSSs. In Fig. 10 we present the relationship between the expected stellar oxygen and sodium abundances $([\mathrm{O} / \mathrm{Fe}],[\mathrm{Na} / \mathrm{Fe}])$ and their absolute $\mathrm{F} 555 \mathrm{~W}$-band magnitudes, calculated using the PARSEC stellar evolutionary models (Bressan et al. 2012) for a metallicity of $Z=0.01$. A clear correlation between luminosities and elemental abundances appears, especially for stars that are brighter than the typical magnitude of the MSTO region. To measure a significant number of BSS magnitudes and chemical abundances, relatively old OCs would be good targets. Since their member stars have already evolved to old ages, there should have been sufficient opportunities for binary mergers to occur. An ideal target is the 6.5 Gyr-old OC NGC 188 (Demarque et al. 1992). Mathieu \& Geller (2009) found that approximately $76 \%$ of its BSSs are members of binary systems, indicating a large fraction of interacting binary stars. 


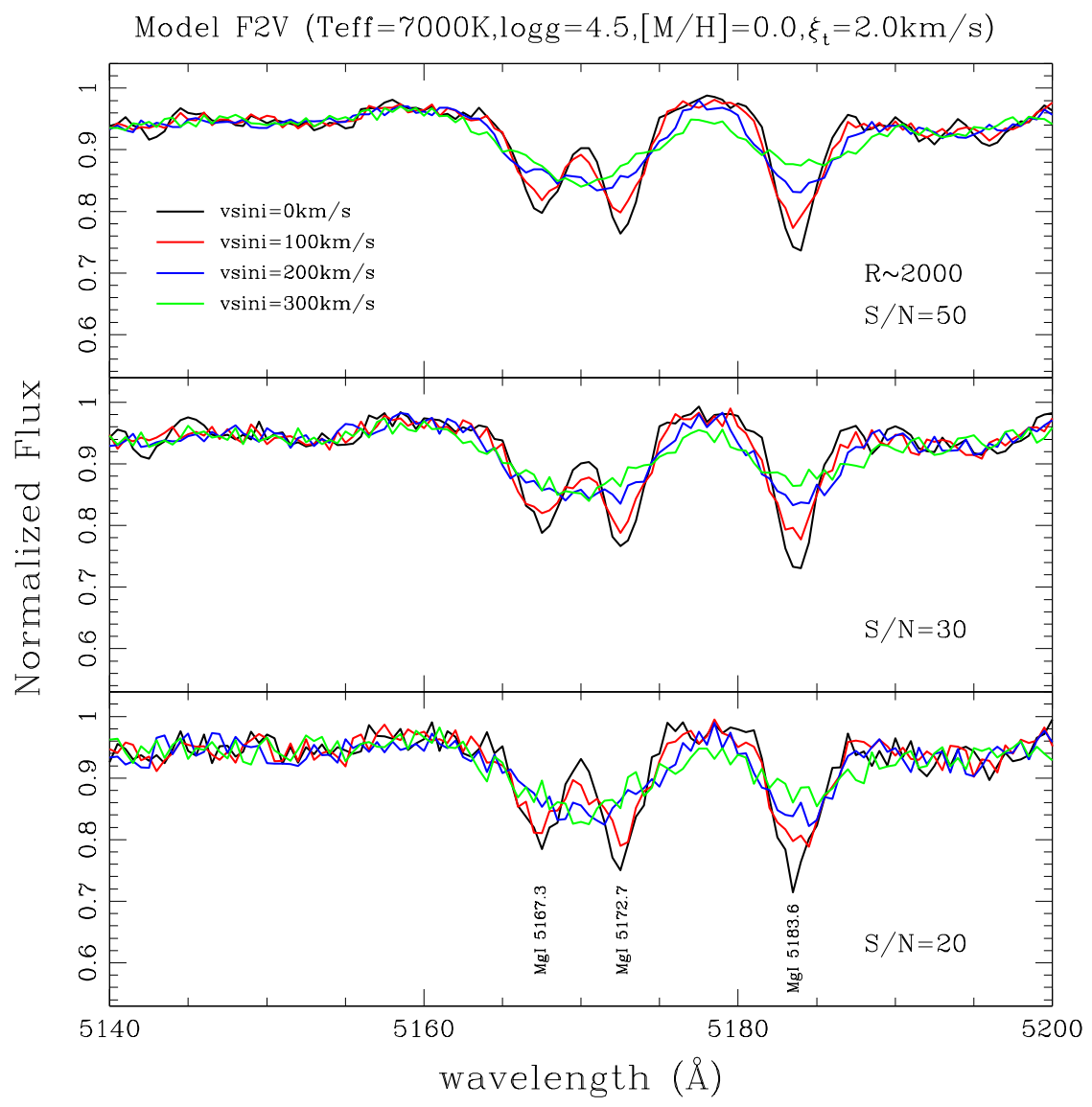

Fig. 9 Simulations of the MgI triplet of an F2V star for different rotation velocities of $0 \mathrm{~km}$ $\mathrm{s}^{-1}$ (black), $100 \mathrm{~km} \mathrm{~s}^{-1}$ (red), $200 \mathrm{~km} \mathrm{~s}^{-1}$ (blue), and 300 (green) $\mathrm{km} \mathrm{s}^{-1}$. The rotation axis is oriented perpendicularly to the line of sight. From top to bottom, the signal-to-noise ratio $(\mathrm{S} / \mathrm{N})=50,30,20$; the adopted spectral resolution is $R \sim 2000$. If the mechanism responsible for the eMSTOs in intermediate-age star clusters is rapid rotation, then it will be detected at the minimum $\mathrm{S} / \mathrm{N}=30$ for most of the lines.

\subsection{Studying Ambient Gas Accretion Through Numerical Simulations}

Significantly more work is required before we can connect young GCs to genuine GCs (which are usually defined as more massive than $10^{5} \mathrm{M}_{\odot}$ - see Fig. 1 -and older than $10 \mathrm{Gyr}$ ). Numerous studies have addressed this issue. In general, YMCs should contain sufficient numbers of member stars initially to survive for a Hubble time (Portegies Zwart et al. 2010). Another key parameter that determines the survival time of YMCs is the slope of its stellar initial mass function (de Grijs \& Parmentier 2007). Current consensus on long-term cluster survival implies that the combination of internal and external cluster dynamics must play a important role (de Grijs 2010$).$

The discovery of young stellar populations in intermediate-age LMC star clusters is exciting. It indicates that star clusters may have access to various channels to form secondary stellar populations 

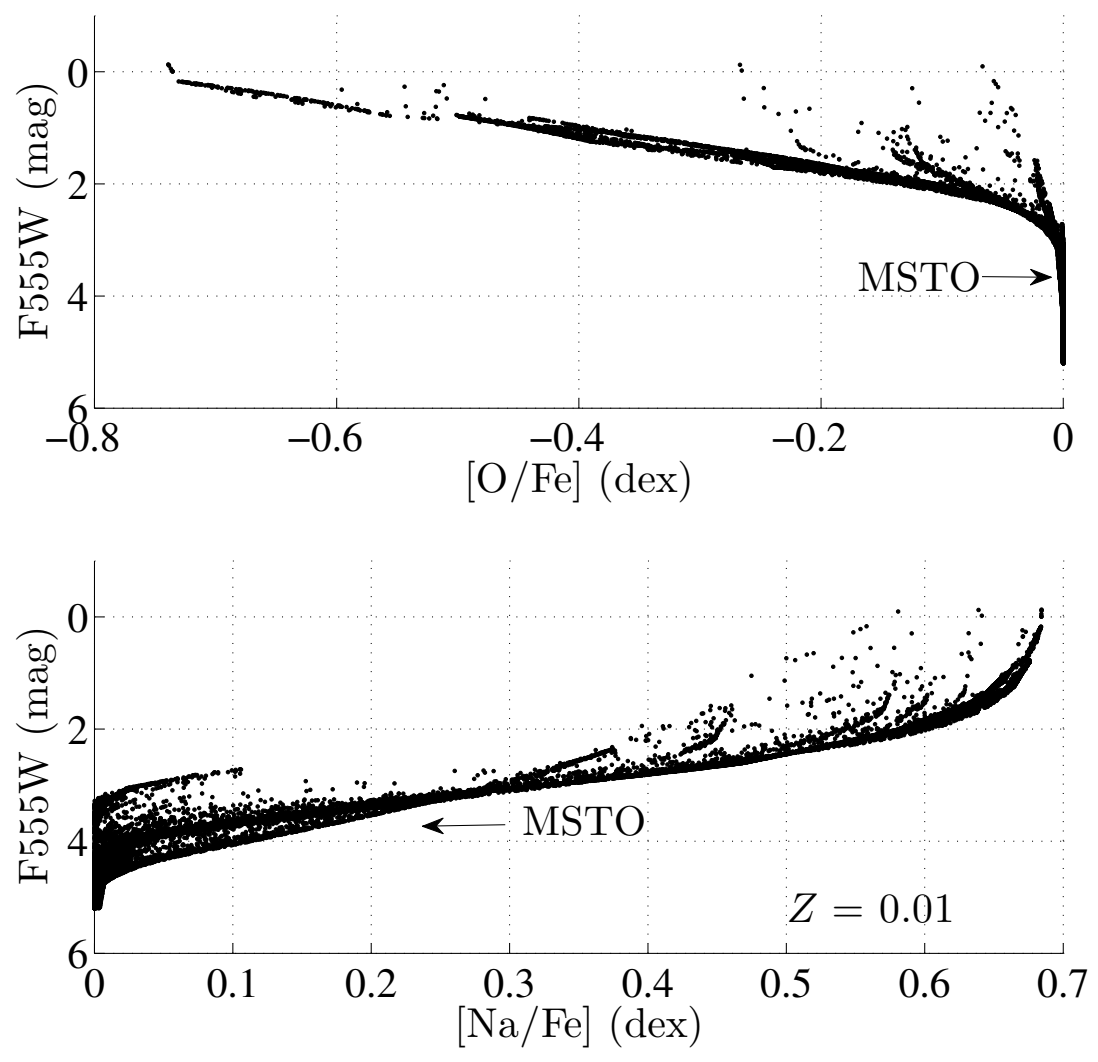

Fig. 10 Stellar F555W magnitude as a function of (top) $[\mathrm{O} / \mathrm{Fe}]$ and (bottom) $[\mathrm{Na} / \mathrm{Fe}]$ abundance, based on Jiang et al. (2014)'s model. The arrows indicate the typical magnitude of the MSTO region. The metallicity adopted is $Z=0.01$.

(Li et al. 2016b). A key difference between the observed secondary stellar populations in these young GCs compared with old stellar populations in genuinely old GCs relates to their dynamics. The young stellar populations in the young clusters are less centrally concentrated than the evolved stars of the first generation. In old GCs the secondary stellar generations are usually more densely distributed than the first-generation stars. Therefore, it is still unclear if the observed secondary stellar generations in these relatively young clusters are similar to the second-generation stars in old GCs.

More evidence for more evolved star clusters (e.g., with ages between $3 \mathrm{Gyr}$ and $10 \mathrm{Gyr}$ ) is indeed required. Unfortunately, there is an age gap between $3 \mathrm{Gyr}$ and $10 \mathrm{Gyr}$ in the LMC cluster sample (Balbinot et al. 2010), so one has to turn to numerical simulations to study this aspect. Li et al. (2016b) proposed that ambient gas accretion may be a possible solution to the observed young stellar populations. They speculated that GMCs are possible sources of accreted gas. If this is correct, then accretion should be a common process for all massive star clusters (irrespective of their ages) that are located in gas-rich galactic disks. However, the nature of the slightly less concentrated spatial distributions of the young stellar populations compared with their first-generation 
RGB counterparts is unresolved. Cabrera-Ziri et al. (2016) argued that the observed features may have been caused by misunderstood issues in field-star decontamination, but it is highly unlikely that field-star contamination could artificially produce such tightly constrained, well-populated stellar populations (see Fig. 6), which also exhibit clearly centrally concentrated radial profiles (see Fig. 7). Additional studies of the dynamics of these younger stellar populations are required: indeed, one needs to combine $N$-body simulations with up-to-date insights into stellar evolution to explore these issues. More accurate models should also place clusters in an external gravitational field, which will affect the 'evaporation' processes in different stellar populations (for more details, see Spitzer 1987). More detailed calculations should also use smoothed-particle hydrodynamics simulations to trace accretion flows between star clusters and GMCs.

\section{SUMMARY}

In this review, we have introduced the community's up-to-date insights into the physics governing stellar populations in star clusters. We have shown that because of initial gas expulsion, most star clusters are not expected to exhibit multiple episodes of star formation. This renders the origin of the common observation of multiple stellar populations in star clusters an intriguing open question. The observational status of stellar populations in star clusters can be summarized as follows:

- For star clusters younger than $\sim 100 \mathrm{Myr}$, no conclusive evidence exists that they may harbor multiple stellar populations or ongoing star formation; no residual gas has been detected in extremely young clusters (Bastian \& Strader 2014).

- The eMSTO morphologies of some YMCs with ages older than 100 Myr (e.g., NGC 1850 and NGC 1856; Milone et al. 2015a; Bastian et al.2016) are inconsistent with the expectations from SSPs. It appears that such eMSTOs are a common feature of intermediate-age star clusters in the LMC and SMC. However, spectroscopic analyses have shown that their member stars do no exhibit abundance anomalies (Mucciarelli et al.|2014).

- The presence of multiple stellar populations in old GCs is irrefutable: both the morphologies of their photometric features and star-to-star chemical abundance variations challenge the SSP scenario.

Various scenarios have been proposed to explain these observed deviations from genuine SSPs. For young and intermediate-age star clusters, age spreads (which favor eSFHs) and rapid stellar rotation (which suggests that clusters are SSPs) are in competition. For old GCs, all scenarios can be classified as either primordial or evolutionary.

We have proposed a number of projects that seem feasible in the near future and which may shed light on our understanding of stellar population problems in star clusters. These include direct measurements of stellar rotation rates in compact star clusters, studies of the elemental abundances of BSSs, and the use of numerical simulations to study gas accretion. Since a number of possible candidate young GCs have been identified in nearby starburst galaxies, employing next-generation telescopes to study these objects will significantly contribute to an improved understanding of the origin of stellar populations in star clusters.

Acknowledgements C. L. is partially supported by a Macquarie Research Fellowship and by Strategic Priority Program 'The Emergence of Cosmological Structures' of the Chinese Academy of Sciences (grant XDB09000000). R. d. G. and L. D. acknowledge research support from the National Natural Science Foundation of China through grants 11073001, 11373010, and 11473037.

\section{References}

Allison, R. J., Goodwin, S. P., Parker, R. J., et al. 2009, ApJ, 700, L99 
Amaro-Seoane, P., Konstantinidis, S., Brem, P., \& Catelan, M. 2013, MNRAS, 435, 809

Anderson, J., Piotto, G., King, I. R., Bedin, L. R., \& Guhathakurta, P. 2009, ApJ, 697, L58

Balbinot, E., Santiago, B. X., Kerber, L. O., Barbuy, B., \& Dias, B. M. S. 2010, MNRAS, 404, 1625

Banerjee, S., \& Kroupa, P. 2015, in: The Origin of Stellar Clusters, ed. S. Stahler (Springer), in press (arXiv:1512.03074)

Bastian, N., \& Goodwin, S. P. 2006, MNRAS, 369, L9

Bastian, N., \& de Mink, S. E. 2009, MNRAS, 398, L11

Bastian, N., Trancho, G., Konstantopoulos, I. S., \& Miller, B. W. 2009, ApJ, 701, 607

Bastian, N., Cabrera-Ziri, I., Davies, B., \& Larsen, S. S. 2013, MNRAS, 436, 2852

Bastian, N., \& Strader, J. 2014, MNRAS, 443, 3594

Bastian, N., Hollyhead, K., \& Cabrera-Ziri, I. 2014, MNRAS, 445, 378

Bastian, N., \& Niederhofer, F. 2015, MNRAS, 448, 1863

Bastian, N., Cabrera-Ziri, I., \& Salaris, M. 2015, MNRAS, 449, 3333

Bastian, N., Niederhofer, F., Kozhurina-Platais, V., et al. 2016, MNRAS, 460, 206

Bate, M. R., Bonnell, I. A., \& Bromm, V. 2003, MNRAS, 339, 577

Baumgardt, H., \& Kroupa, P. 2007, MNRAS, 380, 1589

Bedin, L. R., Piotto, G., Zoccali, M., et al. 2000, A\&A, 363, 159

Bekki, K., \& Mackey, A. D. 2009, MNRAS, 394, 124

Bellini, A., Piotto, G., Bedin, L. R., et al. 2009, A\&A, 507, 1393

Bik, A., Stolte, A., Gennaro, M., et al. 2014, A\&A, 561, A12

Bonnell, I. A., Clark, P., \& Bate, M. R. 2008, MNRAS, 389, 1556

Bragaglia, A., Sneden, C., Carretta, E., et al. 2014, ApJ, 796, 68

Brandl, B., Sams, B. J., Bertoldi, F., et al. 1996, ApJ, 466, 254

Brandt, T. D., \& Huang, C. X. 2015, ApJ, 807, 25

Bressan, A., Marigo, P., Girardi, L., et al. 2012, MNRAS, 427, 127

Brogaard, K., VandenBerg, D. A., Bruntt, H., et al. 2012, A\&A, 543, A106

Brüns, R. C., Kroupa, P., \& Fellhauer, M. 2009, ApJ, 702, 1268

Cabrera-Ziri, I., Bastian, N., Davies, B., Magris, G., Bruzual, F., \& Schweizer, F. 2014, MNRAS, 441, 2754

Cabrera-Ziri, I., Bastian, N., Longmore, S. N., et al. 2015, MNRAS, 448, 2224

Cabrera-Ziri, I., Bastian, N., Hilker, M., et al. 2016, MNRAS, 457, 809

Cabrera-Ziri, I., Niederhofer, F., Bastian, N., et al. 2016, MNRAS, 459, 4218

Cantat-Gaudin, T., Vallenari, A., Zaggia, S., et al. 2014, A\&A, 569, A17

Carney, B. W., Latham, D. W., Laird, J. B., Grant, C. E., \& Morse, J. A. 2001, AJ, 122, 3419

Carretta, E., Bragaglia, A., \& Cacciari, C. 2004a, ApJ, 610, L25

Carretta, E., Gratton, R. G., Bragaglia, A., Bonifacio, P., \& Pasquini, L. 2004b, A\&A, 416, 925

Carretta, E., Bragaglia, A., Gratton, R. G., et al. 2009, A\&A, 505, 117

Carretta, E., Gratton, R. G., Lucatello, S., et al. 2010, ApJ, 722, L1

Carretta, E., Bragaglia, A., Gratton, R. G., et al. 2013, A\&A, 557, A138

Cassisi, S., Salaris, M., Pietrinferni, A., et al. 2008, ApJ, 672, L115

Cayrel, R. 1986, A\&A, 168, 81

Chilingarian, I. V., \& Mamon, G. A. 2008, MNRAS, 385, L83

Cohen, J. G., Persson, S. E., \& Frogel, J. A. 1978, ApJ, 222, 165

Colucci, J. E., \& Bernstein, R. A. 2012, ApJ, 749, 124

Correnti, M., Goudfrooij, P., Puzia, T. H., \& de Mink, S. E. 2015, MNRAS, 450, 3054

Conroy, C., \& Spergel, D. N. 2011, ApJ, 726, 36

D’Antona, F., Bellazzini, M., Caloi, V., et al. 2005, ApJ, 631, 868

D'Antona, F., Di Criscienzo, M., Decressin, T., et al. 2015, MNRAS, 453, 2637

D'Antona, F., Vesperini, E., D'Ercole, A., et al. 2016, MNRAS, 458, 2122 
Davidge, T. J. 2012, ApJ, 761, 155

Decressin, T., Meynet, G., Charbonnel, C., Prantzos, N., \& Ekström, S. 2007, A\&A, 464, 1029

de Grijs, R., \& Parmentier, G. 2007, ChJAA (Chin. J. Astron. Astrophys.), 7, 155

de Grijs, R. 2010, RSPTA, 368, 693

Demarque, P., Green, E. M., \& Guenther, D. B. 1992, AJ, 103, 151

de Mink, S. E., Pols, O. R., Langer, N., \& Izzard, R. G. 2009, A\&A, 507, L1

Denissenkov, P. A., VandenBerg, D. A., Hartwick, F. D. A., et al. 2015, MNRAS, 448, 3314

De Silva, G. M., D’Orazi, V., Melo, C., et al. 2013, MNRAS, 431, 1005

Dieball, A., \& Grebel, E. K. 2000, A\&A, 358, 897

Djorgovski, S., \& Meylan, G. 1994, AJ, 108, 1292

D’Orazi, V., \& Randich, S. 2009, A\&A, 501, 553

Drinkwater, M. J., Gregg, M. D., Hilker, M., et al. 2003, Nature, 423, 519

Duncan, D. K., \& Jones, B. F. 1983, ApJ, 271, 663

Eggen, O. J. 1998, AJ, 116, 284

Ekström, S., Georgy, C., Eggenberger, P., et al. 2012, A\&A, 537, A146

Farias, J. P., Smith, R., Fellhauer, M., et al. 2015, MNRAS, 450, 2451

Faulkner, J., Roxburgh, I. W., \& Strittmatter, P. A. 1968, ApJ, 151, 203

Geisler, D., Villanova, S., Carraro, G., et al. 2012, ApJ, 756, L40

Georgiev, I. Y., Hilker, M., Puzia, T. H., Goudfrooij, P., \& Baumgardt, H. 2009, MNRAS, 396, 1075

Georgy, C., Ekström, S., Granada, A., et al. 2013a, A\&A, 553, A24

Georgy, C., Ekström, S., Eggenberger, P., et al. 2013b, A\&A, 558, A103

Georgy, C., Granada, A., Ekström, S., et al. 2014, A\&A, 566, A21

Girardi, L., Rubele, S., \& Kerber, L. 2009, MNRAS, 394, L74

Girardi, L., Eggenberger, P., \& Miglio, A. 2011, MNRAS, 412, L103

Girardi, L., Goudfrooij, P., Kalirai, J. S., et al. 2013, MNRAS, 431, 3501

Girichidis, P., Federrath, C., Allison, R., Banerjee, R., \& Klessen, R. S. 2012, MNRAS, 420, 3264

Goudfrooij, P., Puzia, T. H., Chandar, R., \& Kozhurina-Platais, V. 2011, ApJ, 737, 4

Goudfrooij, P., Girardi, L., Kozhurina-Platais, V., et al. 2014, ApJ, 797, 35

Goudfrooij, P., Girardi, L., Rosenfield, P., et al. 2015, MNRAS, 450, 1693

Gratton, R. G., Bonifacio, P., Bragaglia, A., et al. 2001, A\&A, 369, 87

Gratton, R., Sneden, C., \& Carretta, E. 2004, ARA\&A, 42, 385

Gratton, R. G., Carretta, E., \& Bragaglia, A. 2012, A\&A Rev., 20, 50

Hansen, C. J., \& Kawaler, S. D. 1994, Stellar Interiors. Physical Principles, Structure, and Evolution, XIII,

445 pp. Springer-Verlag: Berlin, Heidelberg, New York.

Hesser, J. E., \& Bell, R. A. 1980, ApJ, 238, L149

Hills, J. G., \& Day, C. A. 1976, Astrophys. Lett., 17, 87

Hypki, A., \& Giersz, M. 2013, MNRAS, 429, 1221

Hollyhead, K., Bastian, N., Adamo, A., et al. 2015, MNRAS, 449, 1106

Jiang, D., Han, Z., \& Li, L. 2014, ApJ, 789, 88

Johnson, J. A., Ivans, I. I., \& Stetson, P. B. 2006, ApJ, 640, 801

Johnson, C. I., \& Pilachowski, C. A. 2010, ApJ, 722, 1373

Kraft, R. P. 1994, PASP, 106, 553

Krumholz, M. R., \& Matzner, C. D. 2009, ApJ, 703, 1352

Lada, C. J., \& Lada, E. A. 2003, ARA\&A, 41, 57

Lardo, C., Bellazzini, M., Pancino, E., et al. 2011, A\&A, 525, A114

Lee, J.-W. 2010, MNRAS, 405, L36

Lee, Y.-W., Joo, J.-M., Sohn, Y.-J., et al. 1999, Nature, 402, 55

Lee, Y.-W., Han, S.-I., Joo, S.-J., et al. 2013, ApJ, 778, L13 
Li, C., de Grijs, R., \& Deng, L. 2013, MNRAS, 436, 149

Li, C., de Grijs, R., \& Deng, L. 2014a, ApJ, 784, 157

Li, C., de Grijs, R., \& Deng, L. 2014b, Nature, 516, 367

Li, C., de Grijs, R., Deng, L., et al. 2014c, ApJ, 790, 35

Li, C., Not-so-simple stellar populations in massive star clusters. 2015, Ph.D. Thesis, Peking University, China

Li, C., de Grijs, R., Bastian, N., Deng, L., Niederhofer, F., \& Zhang, C. 2016a, MNRAS, 461, 3212

Li, C., de Grijs, R., Deng, L., Geller, A. M., Xin, Y., Hu, Y., \& Faucher-Giguère, C. 2016b, Nature, 529, 502

Lima, E. F., Bica, E., Bonatto, C., \& Saito, R. K. 2014, A\&A, 568, A16

Longmore, S. N., Kruijssen, J. M. D., Bastian, N., et al. 2014, Protostars and Planets VI, 291

Mackey, A. D., \& Broby Nielsen, P. 2007, MNRAS, 379, 151

Mackey, A. D., Broby Nielsen, P., Ferguson, A. M. N., \& Richardson, J. C. 2008, ApJ, 681, L17

Maeder, A., \& Meynet, G. 2000, ARA\&A, 38, 143

Marino, A. F., Milone, A. P., Piotto, G., et al. 2009, A\&A, 505, 1099

Marino, A. F., Milone, A. P., Przybilla, N., et al. 2014, MNRAS, 437, 1609

Mathieu, R. D., \& Geller, A. M. 2009, Nature, 462, 1032

McCrea, W. H. 1964, MNRAS, 128, 147

McLaughlin, D. E., \& Fall, S. M. 2008, ApJ, 679, 1272

McNally, D. 1965, The Observatory, 85, 166

Mestel, L., \& Spruit, H. C. 1987, MNRAS, 226, 57

Milone, A. P., Bedin, L. R., Piotto, G., \& Anderson, J. 2009, A\&A, 497, 755

Milone, A. P., Piotto, G., Bedin, L. R., et al. 2012, ApJ, 744, 58

Milone, A. P., Bedin, L. R., Piotto, G., et al. 2015a, MNRAS, 450, 3750

Milone, A. P., Marino, A. F., Piotto, G., et al. 2015b, MNRAS, 447, 927

Milone, A. P., Marino, A. F., D’Antona, F., et al. 2016, MNRAS, 458, 4368

Moeckel, N., \& Burkert, A. 2015, ApJ, 807, 67

Mucciarelli, A., Dalessandro, E., Ferraro, F. R., Origlia, L., \& Lanzoni, B. 2014, ApJ, 793, L6

Naiman, J. P., Ramirez-Ruiz, E., \& Lin, D. N. C. 2011, ApJ, 735, 25

Niederhofer, F., Bastian, N., Kozhurina-Platais, V., et al. 2016, A\&A, 586, 148

Niederhofer, F., Hilker, M., Bastian, N., \& Silva-Villa, E. 2015, A\&A, 575, A62

Nieva, M.-F., \& Simón-Díaz, S. 2011, A\&A, 532, A2

Norris, J., \& Smith, G. H. 1985, AJ, 90, 2526

Norris, J. E., \& Da Costa, G. S. 1995, ApJ, 447, 680

Norris, M. A., Kannappan, S. J., Forbes, D. A., et al. 2014, MNRAS, 443, 1151

Nucci, M. C., \& Busso, M. 2014, ApJ, 787, 141

Palla, F., \& Stahler, S. W. 2000, ApJ, 540, 255

Palla, F., Randich, S., Pavlenko, Y. V., Flaccomio, E., \& Pallavicini, R. 2007, ApJ, 659, L41

Parmentier, G., Jehin, E., Magain, P., et al. 1999, A\&A, 352, 138

Penny, A. J., Smith, G. H., \& Churchill, C. W. 1992, MNRAS, 257, 89

Piotto, G., Bedin, L. R., Anderson, J., King, I. R., Cassisi, S., Milone, A. P., Villanova, S., Pietrinferni, A., \&

Renzini, A. 2007, ApJ, 661, L53

Piotto, G., Milone, A. P., Marino, A. F., et al. 2013, ApJ, 775, 15

Piotto, G., Milone, A. P., Bedin, L. R., et al. 2015, AJ, 149, 91

Portegies Zwart, S. F., McMillan, S. L. W., \& Gieles, M. 2010, ARA\&A, 48, 431

Royer, F., Zorec, J., \& Gómez, A. E. 2007, A\&A, 463, 671

Rubele, S., Kerber, L., \& Girardi, L. 2010, MNRAS, 403, 1156

Rubele, S., Girardi, L., Kozhurina-Platais, V., Goudfrooij, P., \& Kerber, L. 2011, MNRAS, 414, 2204

Sacco, G. G., Randich, S., Franciosini, E., Pallavicini, R., \& Palla, F. 2007, A\&A, 462, L23

Schatzman, E. 1962, Annales d'Astrophysique, 25, 18 
Shen, Z.-X., Jones, B., Lin, D. N. C., Liu, X.-W., \& Li, S.-L. 2005, ApJ, 635, 608

Shetrone, M. D. 1996, AJ, 112, 1517

Shustov, B. M., \& Wiebe, D. S. 2000, MNRAS, 319, 1047

Smith, G. H. 1983, AJ, 88, 410

Smith, G. H. 1988, AJ, 95, 755

Smith, G. H. 2002, PASP, 114, 1097

Smith, G. H., \& Briley, M. M. 2006, PASP, 118, 740

Smith, L. J., Westmoquette, M. S., Gallagher, J. S., et al. 2006, MNRAS, 370, 513

Soderblom, D. R. 2010, ARA\&A, 48, 581

Spitzer, L. 1987, Princeton, NJ, Princeton University Press.

Sweigart, A. V., \& Mengel, J. G. 1979, ApJ, 229, 624

Ting, Y.-S., De Silva, G. M., Freeman, K. C., \& Parker, S. J. 2012, MNRAS, 427, 882

Valcarce, A. A. R., \& Catelan, M. 2011, A\&A, 533, A120

Ventura, P., \& D’ Antona, F. 2009, A\&A, 499, 835

Villanova, S., Piotto, G., King, I. R., et al. 2007, ApJ, 663, 296

Wu, X. H., Li, C., de Grijs, R., \& Deng, L. 2016, ApJL, 826, 14

Yang, W., Bi, S., Meng, X., \& Liu, Z. 2013, ApJ, 776, 112

Yong, D., Grundahl, F., Lambert, D. L., Nissen, P. E., \& Shetrone, M. D. 2003, A\&A, 402, 985

Yong, D., Roederer, I. U., Grundahl, F., et al. 2014, MNRAS, 441, 3396

Yusof, N., Hirschi, R., Meynet, G., et al. 2013, MNRAS, 433, 1114 